\title{
Renewable cellulosic nanocomposites for food packaging to avoid fossil fuel plastic pollution: a review
}

\author{
Umair Qasim ${ }^{1} \cdot$ Ahmed I. Osman $^{2}$ (1) - Ala'a H. Al-Muhtaseb ${ }^{3} \cdot$ Charlie Farrell $^{4,5} \cdot$ Mohammed Al-Abri $^{3,6} \cdot$ Muzaffar Ali $^{1}$. \\ Dai-Viet N. Vo ${ }^{7}$. Farrukh Jamil ${ }^{1}$. David W. Rooney ${ }^{2}$
}

Received: 19 May 2020 / Accepted: 5 September 2020 / Published online: 24 September 2020

(C) The Author(s) 2020

\begin{abstract}
The extensive use of petroleum-based synthetic and non-biodegradable materials for packaging applications has caused severe environmental damage. The rising demand for sustainable packaging materials has encouraged scientists to explore abundant unconventional materials. For instance, cellulose, extracted from lignocellulosic biomass, has gained attention owing to its ecological and biodegradable nature. This article reviews the extraction of cellulose nanoparticles from conventional and non-conventional lignocellulosic biomass, and the preparation of cellulosic nanocomposites for food packaging. Cellulosic nanocomposites exhibit exceptional mechanical, biodegradation, optical and barrier properties, which are attributed to the nanoscale structure and the high specific surface area, of $533 \mathrm{~m}^{2} \mathrm{~g}^{-1}$, of cellulose. The mechanical properties of composites improve with the content of cellulose nanoparticles, yet an excessive amount induces agglomeration and, in turn, poor mechanical properties. Addition of cellulose nanoparticles increases tensile properties by about $42 \%$. Barrier properties of the composites are reinforced by cellulose nanoparticles; for instance, the water vapor permeability decreased by $28 \%$ in the presence of $5 \mathrm{wt} \%$ cellulose nanoparticles. Moreover, $1 \mathrm{wt} \%$ addition of filler decreased the oxygen transmission rate by $21 \%$. We also discuss the eco-design process, designing principles and challenges.
\end{abstract}

Keywords Biopolymers $\cdot$ Lignocellulosic biomass $\cdot$ Cellulose nanoparticles $\cdot$ Cellulosic nanocomposites $\cdot$ Food packaging $\cdot$ Sustainable packaging

Umair Qasim and Ahmed I. Osman have contributed equally to this work.

Umair Qasim

umairqasimm@gmail.com

Ahmed I. Osman

aosmanahmed01@qub.ac.uk; a.osman@qub.ac.uk

$\triangle$ Ala'a H. Al-Muhtaseb

muhtaseb@squ.edu.om

$\triangle$ Farrukh Jamil

fjamil@ cuilahore.edu.pk; fjamil44@gmail.com

Charlie Farrell

charlie.farrell@swc.ac.uk; cfarrell13@qub.ac.uk

Mohammed Al-Abri

alabri@squ.edu.om

Muzaffar Ali

muzaffaraliuetr@gmail.com

Dai-Viet N. Vo

vndviet@ntt.edu.vn

David W. Rooney

d.rooney@qub.ac.uk
Department of Chemical Engineering, COMSATS University Islamabad-Lahore Campus, Lahore, Pakistan

2 School of Chemistry and Chemical Engineering, Queen's University Belfast, David Keir Building, Stranmillis Road, Belfast, Northern Ireland BT9 5AG, UK

3 Department of Petroleum and Chemical Engineering, College of Engineering, Sultan Qaboos University, 123 Muscat, Oman

4 South West College, Cookstown, Co. Tyrone, Northern Ireland BT80 8DN, UK

5 School of Mechanical and Aerospace Engineering, Queen's University Belfast, Stranmillis Road, Belfast, Northern Ireland BT9 5AH, UK

6 Nanotechnology Research Centre, Sultan Qaboos University, Muscat, Oman

7 Center of Excellence for Green Energy and Environmental Nanomaterials (CE@GrEEN), Nguyen Tat Thanh University, 300A Nguyen Tat Thanh, District 4, Ho Chi Minh City 755414, Vietnam 


\section{Introduction}

Packaging is used to preserve food quality and provide hygiene safety for the consumer (Zhang et al. 2014; Wang et al. 2019; Gaikwad et al. 2019). It helps in the handling, transportation, and storage by protecting food products from physical (crushing, abrasions, and shocks), chemical (ultraviolet radiations), and biological (microorganism) damage (Al-Tayyar et al. 2020a; Youssef et al. 2019). It also delivers essential information about the ingredients, product characteristics, and nutritional content. Additionally, the packaging is considered as a global marketing tool as in 2015 , around $\$ 839 \times 10^{9}$ was the total revenue generated by the food packaging industry, which is expected to peak at a rate of $3.5 \%$ between 2015 and 2020, reaching a value of $\$ 998$ billion in 2020 . Almost $85 \%$ of the packaging sector is occupied by food and beverages, followed by pharmaceutical products, constituting 10\% (Gaikwad et al. 2018). The paper packaging industry currently reaches 7.6 million tons, followed by plastic packaging (6.8 Million tons) (Bharimalla et al. 2019).

Over the past decades, the food industry has been using petroleum-based plastic materials owing to their attractive properties, such as flexibility, safety, versatility, and low cost (Gutiérrez and Alvarez 2017a; Padervand et al. 2020). Irrespective of these properties, major drawbacks are accompanying these synthetic materials, such as non-biodegradability, disposal, and recycling (Tice 2003; Khalil et al. 2017; Tofa et al. 2019). In addition, environmental issues, high prices, and attrition of petroleum resources are some of the concerning factors of these materials. With the advancement in material science technologies, consumer behavior changes and this had led to the emergence of sustainable packaging materials, which serves as an alternative to conventional synthetic materials (Gutiérrez and
Alvarez 2017b; Gaikwad et al. 2020). Ongoing research aims to meet the environmental legislation and consumer demands for innovative and sustainable food packaging (Merino et al. 2019a, 2019). In comparison with the petroleum-based conventional materials, biopolymers (cellulose, starch, chitosan) are gaining increased attention because of their renewable \& cheaper nature (compared to the former) and could be derived from agricultural and marine sources (Rai et al. 2019; El-Sayed et al. 2020).

Biopolymers are naturally occurring polymers that are generally described as polymers that originated from renewable resources (Fig. 1). Biopolymers are considered as ecofriendly materials as well as biodegradable. Among innumerable applications, food packaging is the most attractive application of biopolymers (Youssef and El-Sayed 2018). Properties of some important biopolymers are presented in Table 1.

Among various biopolymers, cellulose is an abundant, sustainable, and the most prosperous bio-based polymer. Millimeter-sized strings made up of continuously little microfibers, and microfibers that contain microfibrils of nanometer measurements formed the fundamental structure of cellulose. Cellulose is anticipated to be less expensive, non-toxic, and has a remarkable strength-to-weight ratio compelling to be used extensively in the packaging industry (Swain and Mohanty 2018). Figure 2 shows the production of biodegradable food packaging from cellulosic materials derived from lignocellulosic biomass as a promising and attractive approach to solve the plastic food packaging problem.

Lignocellulosic biomass contains cellulose (crystalline region), hemicellulose, lignin (amorphous region), and other non-cellulosic components such as pectin and waxes (Osman et al. 2020; Osman et al. 2019; Chen et al. 2019). In the 1980s, cellulose nanoparticles were found as a novel type of plant-based polymer, which includes cellulose
Fig. 1 Biopolymers, naturally occurring renewable and abundant polymers, are classified into three groups based on their origin. Some biopolymers, such as starch, cellulose, chitosan, and alginates, can be extracted from biomass through various treatments (chemical, mechanical, or biological). Similarly, biopolymers, including pullulan, curdlan and bacterial cellulose, can be derived from microorganisms. A new class of biopolymers, such as polyamides, polylactic acid, and carboxylic acids, can be synthetically prepared in laboratories

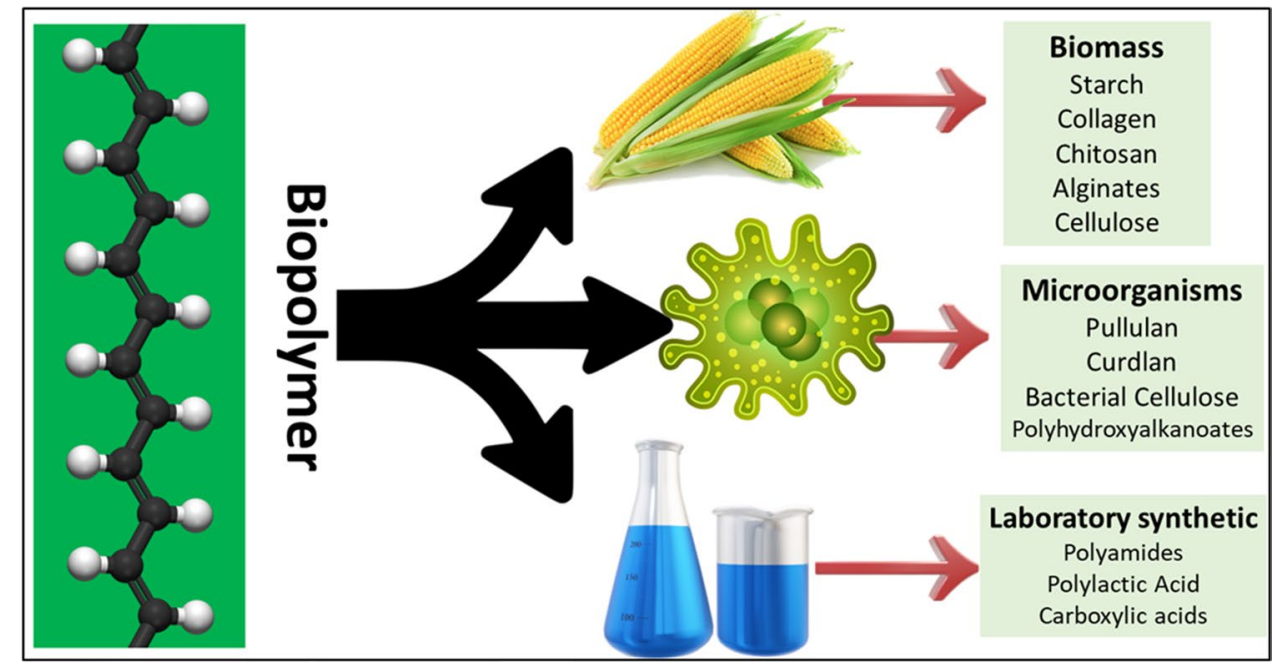


Table 1 Composition and properties of some common biopolymers that are used in food packaging applications

\begin{tabular}{|c|c|c|c|}
\hline Polysaccharide & Composition & Properties & References \\
\hline Alginate & Mannuronic glucuronic acid & $\begin{array}{l}\text { Brittle } \\
\text { Biodegradable } \\
\text { High water permeability } \\
\text { Cross-link with calcium }\end{array}$ & Galgano (2015) and Campos et al. (2011) \\
\hline Chitin & $N$-acetylglucosamine & $\begin{array}{l}\text { Transparent } \\
\text { Non-toxic } \\
\text { Biodegradable } \\
\text { Biocompatible } \\
\text { Antifungal and antibacterial }\end{array}$ & Ferreira et al. (2016) \\
\hline Cellulose & Glucose & $\begin{array}{l}\text { Biodegradable } \\
\text { Transparent } \\
\text { Sensitive to water } \\
\text { Good mechanical properties }\end{array}$ & $\begin{array}{l}\text { Campos et al. (2011) and Cruz-Romero and Kerry } \\
\text { (2008) }\end{array}$ \\
\hline Chitosan & D-Glucosamine $N$-acetyl-D-glucosamine & $\begin{array}{l}\text { Brittle } \\
\text { Biodegradable } \\
\text { Antibacterial and antifungal } \\
\text { Non-toxic } \\
\text { Barrier to gases }\end{array}$ & $\begin{array}{l}\text { Cazon and Vazquez (2020) and Morin-Crini et al. } \\
\text { (2019) }\end{array}$ \\
\hline Carrageenan & Galactose & $\begin{array}{l}\text { Fragile } \\
\text { Ductile } \\
\text { Biodegradable }\end{array}$ & Campos et al. (2011) \\
\hline FucoPol & $\begin{array}{l}\text { Fucose, galactose, glucose } \\
\text { acetate, succinate, pyruvate }\end{array}$ & $\begin{array}{l}\text { Transparent } \\
\text { Biodegradable } \\
\text { High gas barrier } \\
\text { Poor water resistance }\end{array}$ & Ferreira et al. (2016) \\
\hline Gellan gum & $\begin{array}{l}\text { Glucose } \\
\text { Rhamnose } \\
\text { Glucuronic acid }\end{array}$ & $\begin{array}{l}\text { Edible } \\
\text { Lipid barrier } \\
\text { Biodegradable } \\
\text { Good tensile strength } \\
\text { Excellent gas barrier }\end{array}$ & Ferreira et al. (2016) and Ferreira et al. (2014) \\
\hline Galactomannans & $\begin{array}{l}\text { Galactose } \\
\text { Mannose }\end{array}$ & $\begin{array}{l}\text { Edible } \\
\text { Biodegradable } \\
\text { Semi-permeable barrier to gas }\end{array}$ & Galgano (2015) and Cerqueira et al. (2011) \\
\hline Pullulan & Maltotriose (three glucose) & $\begin{array}{l}\text { Edible } \\
\text { Transparent } \\
\text { Biodegradable } \\
\text { Heat sealable } \\
\text { High water solubility } \\
\text { Barrier to oxygen } \\
\text { Oil resistant } \\
\text { Grease resistant }\end{array}$ & Cheng et al. (2011) and Zhang et al. (2013) \\
\hline Xanthan gum & $\begin{array}{l}\text { Glucose, mannose, glucuronic acid, } \\
\text { acetate, pyruvate }\end{array}$ & $\begin{array}{l}\text { Edible } \\
\text { Biodegradable }\end{array}$ & Quoc et al. (2015) \\
\hline
\end{tabular}

fibrils and crystals in the nanodimensions. The longitudinal measurement goes from a couple of 10's of nanometers to a few microns, and horizontal components extend from 5 to $20 \mathrm{~nm}$ (Zinge and Kandasubramanian 2020). Cellulose nanoparticles are categorized as cellulose nanocrystals, cellulose nanofibers, and bacterial cellulose (de Amorim et al. 2020). Acid hydrolysis treatment on wood or any cellulosecontaining materials results in cellulose nanocrystals with the width ranging from 3 to $20 \mathrm{~nm}$ and the length of around 50-500 nm, respectively.

Physical treatments, with or without chemical treatments, could be used to obtain linear or branched chains cellulose nanofibers of width and length ranging from 4 to $50 \mathrm{~nm}$ and $500 \mathrm{~nm}$, respectively (Thakur 2013). Bacterial cellulose is chemically similar to plant cellulose and is formed according to the bottom-up method, in which cellulose in the form of nanofibrils is produced by the bacteria, which gathers as a ribbon with the width around 70-80 nm (Pecoraro et al. 2007). Bacterial cellulose can also be synthesized in the pure form, free from the noncellulosic components (hemicellulose and lignin), thus reducing the cost and usage of chemical reagents for the removal of lignin and hemicellulose components (de Amorim et al. 2020; Duarte et al. 2015). 

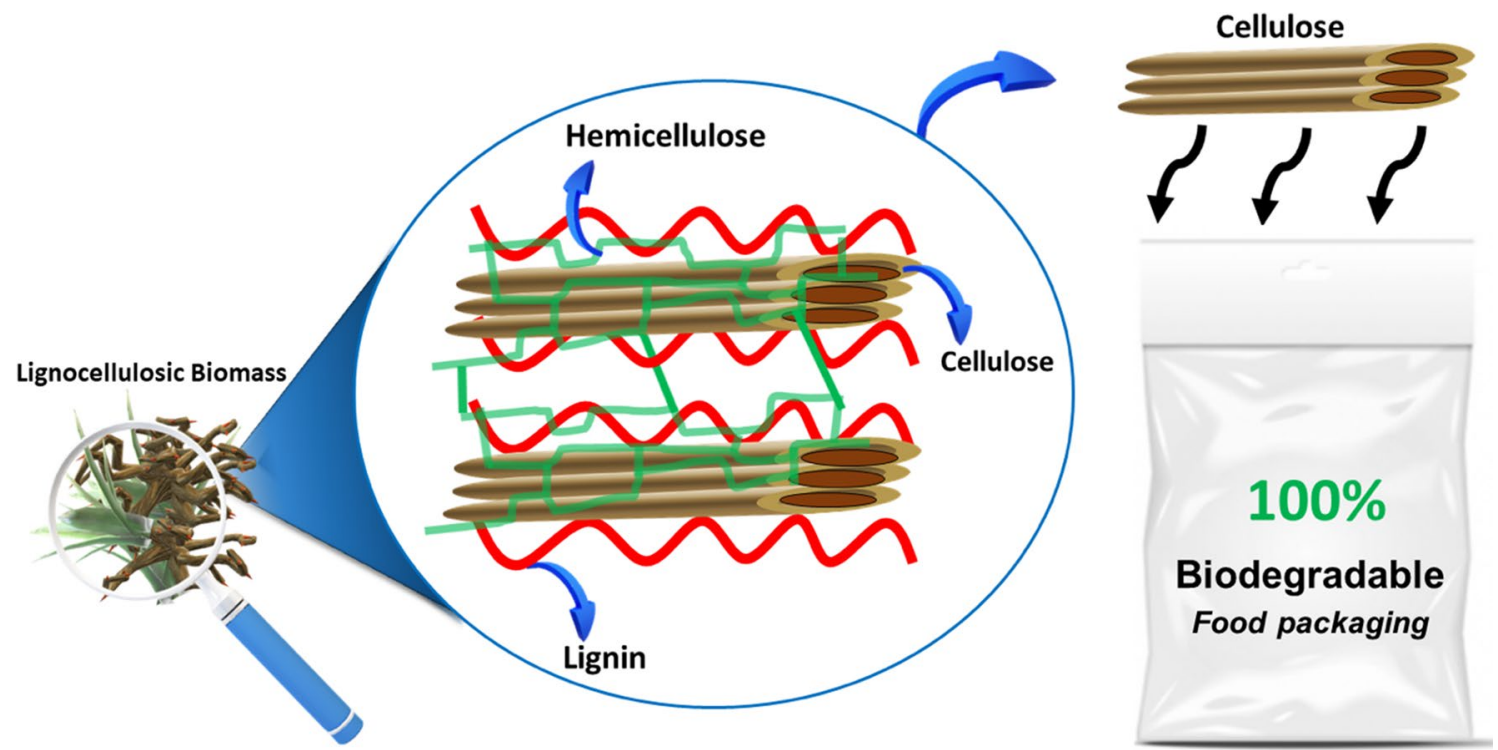

Fig. 2 Structural composition of lignocellulosic biomass, which is predominantly composed of cellulose, hemicellulose, and lignin. Cellulose is the most abundant component and can be extracted and used for the production of biodegradable food packaging

Besides the properties mentioned above of cellulose, the use of cellulose is still restricted due to the poor barrier and mechanical properties, thermal stability, and water absorption ability (Cazon and Vazquez 2020). These shortcomings hinder the use of cellulose in contrast to orthodox synthetic materials. A probable solution to this problem is the use of cellulose as a filler to make composite materials. Nanocomposites are multi-phase materials, in which the dispersed phase constituents possess at least one dimension under $100 \mathrm{~nm}$ or less (Al-Tayyar et al. 2020b). Cellulose nanoparticles display better stiffness with a tensile modulus of $134 \mathrm{GPa}$ and the tensile strength in the range of $0.8-10 \mathrm{GPa}$ (Azeredo et al. 2010; Cao et al. 2008). Kumar et al. (Kumar et al. 2019) prepared green nanocomposite films based on polylactic acid and cellulose nanofibers using the solvent casting method for packaging application. Frone et al. (2019) prepared aliphatic polyester-based biodegradable films containing nanocellulose extracted from plum shells agricultural waste. Tang et al. (2017) developed biodegradable scaffolds for their use in tissue engineering.

Cellulose nanoparticles can be considered as a pioneering approach for the development of sustainable packaging with enriched features. In terms of designing, an effectual design may consist of quantitative and qualitative functioning of the product (ecological packaging) throughout its entire life cycle. Designing process, for the isolation of cellulose nanoparticles, will certify the quality of product and the requirements of the product packaging, for instance; size, thickness, safety and stress levels, and ergonomics, as well as its quantitative life cycle assessment and cost. Therefore, the principal role attributed to the designing process is to define the possibilities, limitations, and suitability of cellulose nanoparticles for sustainable packaging development (Khalil et al. 2016).

In view of the cutting-edge impact of cellulose nanoparticles in food packaging, we divulge the audience with an in-depth view of the current research surrounding cellulose and cellulose nanoparticles. Detail sketch on the pretreatments, properties of cellulose nanoparticles, nanocomposites processing techniques with a special focus on food packaging applications has been reviewed. Finally, the need for process designing (for cellulose nanoparticles), as well as the principles, importance, and the challenges in sustainable food packaging, is also discussed in this work.

\section{Isolation of the cellulose nanoparticles}

Extraction of cellulose-enriched materials, having nanoscale dimensions (1-100 nm), from lignocellulosic biomass is the objective of international research. Lignocellulosic biomass forms a complex and compact hetero-matrix structure (Fig. 3) (Xiong et al. 2018). The physicochemical interactions among cellulose, hemicellulose, and lignin are responsible for shielding the cellulose from isolation (Hassan et al. 2020; Zhong et al. 2020). The multistage procedure for the isolation of cellulose nanoparticles from biomass is acquired in two steps. The fragmentation of lignocellulosic material to recover cellulose (step 1) and the treatment of cellulose to 


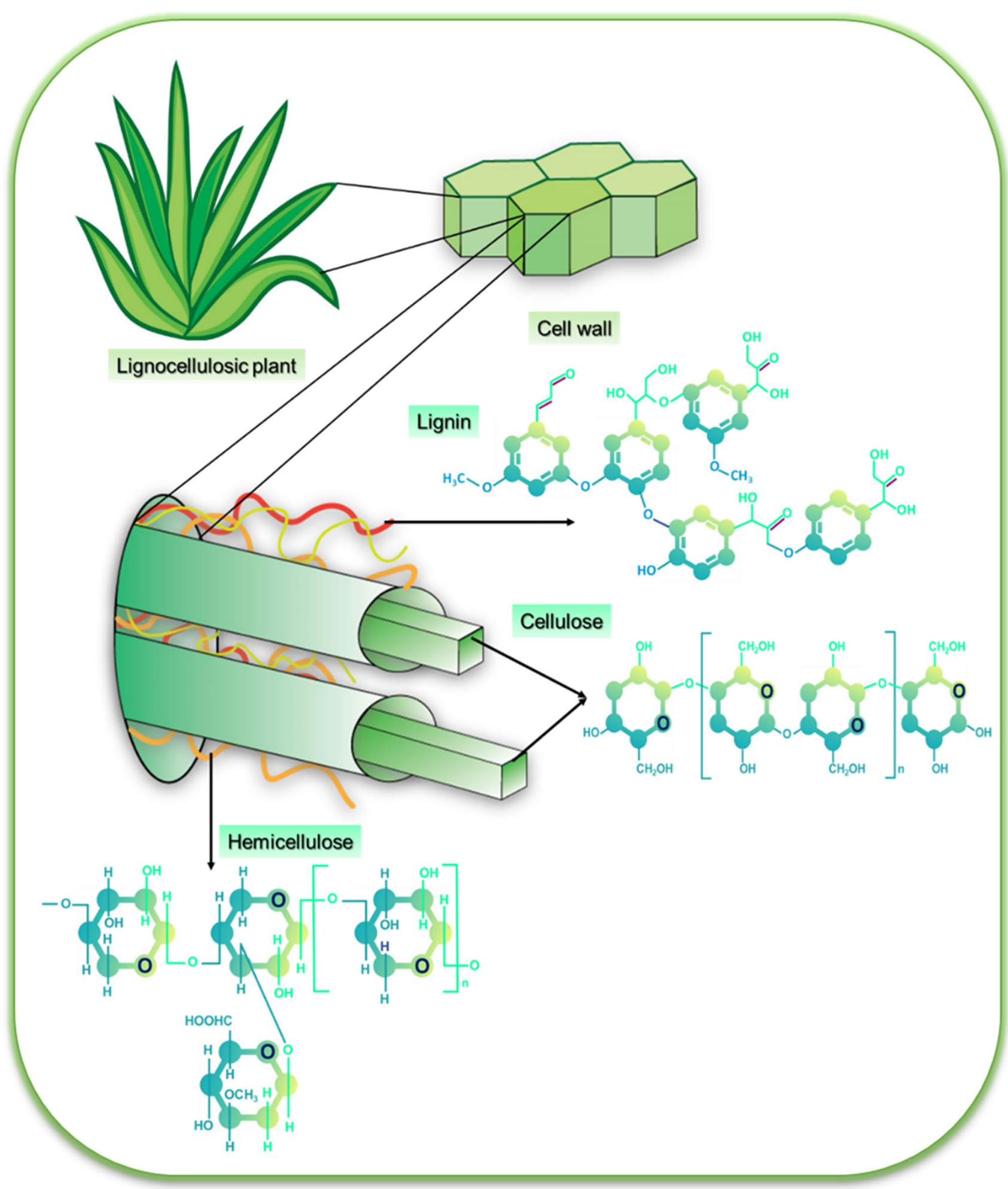

Fig. 3 Cell wall structure of the lignocellulosic plant, which is composed of three oxygen-containing high molecular weight biopolymers: cellulose $\left(\mathrm{C}_{6} \mathrm{H}_{10} \mathrm{O}_{5}\right)_{\mathrm{n}}$, hemicellulose $\left(\mathrm{C}_{5} \mathrm{H}_{8} \mathrm{O}_{4}\right)_{\mathrm{m}}$, and lignin $\left(\mathrm{C}_{9} \mathrm{H}_{10} \mathrm{O}_{3}\left(\mathrm{OCH}_{3}\right)_{0.9-1.7}\right)_{\mathrm{n}}$. These components combined to form a natural fibril-based composite in which hemicellulose acts as a compatibilizer between cellulose and lignin, while lignin protects the cellulose by blocking the bacterial attacks 
get the nanoscale dimension of cellulose (step 2) are shown in Fig. 4 (Sfiligoj Smole et al. 2019; Zhong et al. 2019).

Mechanical techniques, such as micro-fluidization, highintensity ultra-sonication, grinding, and high-pressure homogenization, generate nanofibrillated cellulose by defibrillation (Thomas et al. 2018). Kanoth et al. (2015) ground coir with a mechanical grinder, followed by a chemical process and synthesized 18-20-nm-diameter nanofibrillated cellulose. Stelte and Sanadi fibrillated hardwood and softwood pulps to get the nanofibers by refining and homogenizing at $50 \mathrm{Mpa}$ (Stelte and Sanadi 2009). Wang et al. (2015) studied the isolation of cellulose nanoparticles employing highpressure homogenization. The produced cellulose nanoparticles had a mean particle diameter of 5-20 nm. Xie et al. (2018) reported that balls made of cerium-doped zirconia of diameter $0.4-0.6 \mathrm{~mm}$ were used to prepare the cellulose nanofibers spanning in diameter of 90-110 nm.

Wheat straw was studied as a source of cellulose by several authors. It was revealed that the crystals obtained after the acid hydrolysis of wheat straw are little rods of 150-300 nm in length, 5-nanometer width and an aspect ratio (length to diameter ratio) of 50 (Helbert et al. 1996). Alemdar and Sain used wheat straw and soy hulls to synthesize cellulose nanofibers. The researchers applied chemomechanical approaches and obtained particles with a length of several thousand nanometers and diameter in the range of 10-80 nm (Alemdar and Sain 2008). Similarly, Fatah et al. (2014) applied the chemo-mechanical method on oil palm empty fruit bunch for the extraction of cellulose nanofibers with a diameter ranging from 5 to $10 \mathrm{~nm}$ and observed a decrease in the crystallinity. Chen et al. (2011a) used a chemical ultrasonic technique on four different lignocellulosic fibers (wood, bamboo, wheat straw, and flax fibers) for the extraction of cellulose nanofibers. Nanofibers were successfully extracted from wheat straw, wood, and bamboo with a diameter of $10-40 \mathrm{~nm}$. Acid hydrolysis treatment was employed for the preparation of cellulose nanocrystals from ramie cellulose, and the prepared cellulose nanocrystals had $85.4 \pm 25.3 \mathrm{~nm}$ diameter and $538.5 \pm 125.3 \mathrm{~nm}$ (average) length (Lu et al. 2006). Similarly, cellulose nanocrystals were prepared by acid hydrolysis of flax fibers and the obtained cellulose nanocrystals were slender rods with an average length of $327 \pm 108 \mathrm{~nm}$ and the diameter of $21 \pm 7 \mathrm{~nm}$ (Cao et al. 2007).

Costa et al. (2015) initially alkali-bleached corn husk, followed by the hydrolysis with $64 \%$ sulfuric acid at $4{ }^{\circ} \mathrm{C}$ for 15-60 min and isolated cellulose nanocrystals. Cellulose nanocrystals were also extracted from sisal fibers by combined bleaching and hydrolysis at $60{ }^{\circ} \mathrm{C}$ for about $40 \mathrm{~min}$. Cellulose nanofibers were successfully prepared from water hyacinth cellulose via alkaline-bleaching and sodium chlorite treatment. According to the result, obtained particles showed a diameter in the range between 20 and $100 \mathrm{~nm}$ (Marimuthu and Atmakuru 2015). Mandarin residue, left by citrus juice processing and global production around 8-20 million tons per year, was used to obtain cellulose nanofibers of 2-3 nm thickness (Hiasa et al. 2014). The chardonnay grape skin underwent organic extraction; acid and base dissolution; and basic and acidic oxidation to yield $16.4 \%$ cellulose nanocrystals with high cellulose crystallinity (59.4\%)
Fig. 4 Cellulose nanoparticles can be isolated via two methods. The chemical method results in the isolation of cellulose nanocrystals, whereas the mechanical method isolates cellulose nanofibers. Both types of cellulose differ in terms of their specific surface area (Sofla et al. 2016)

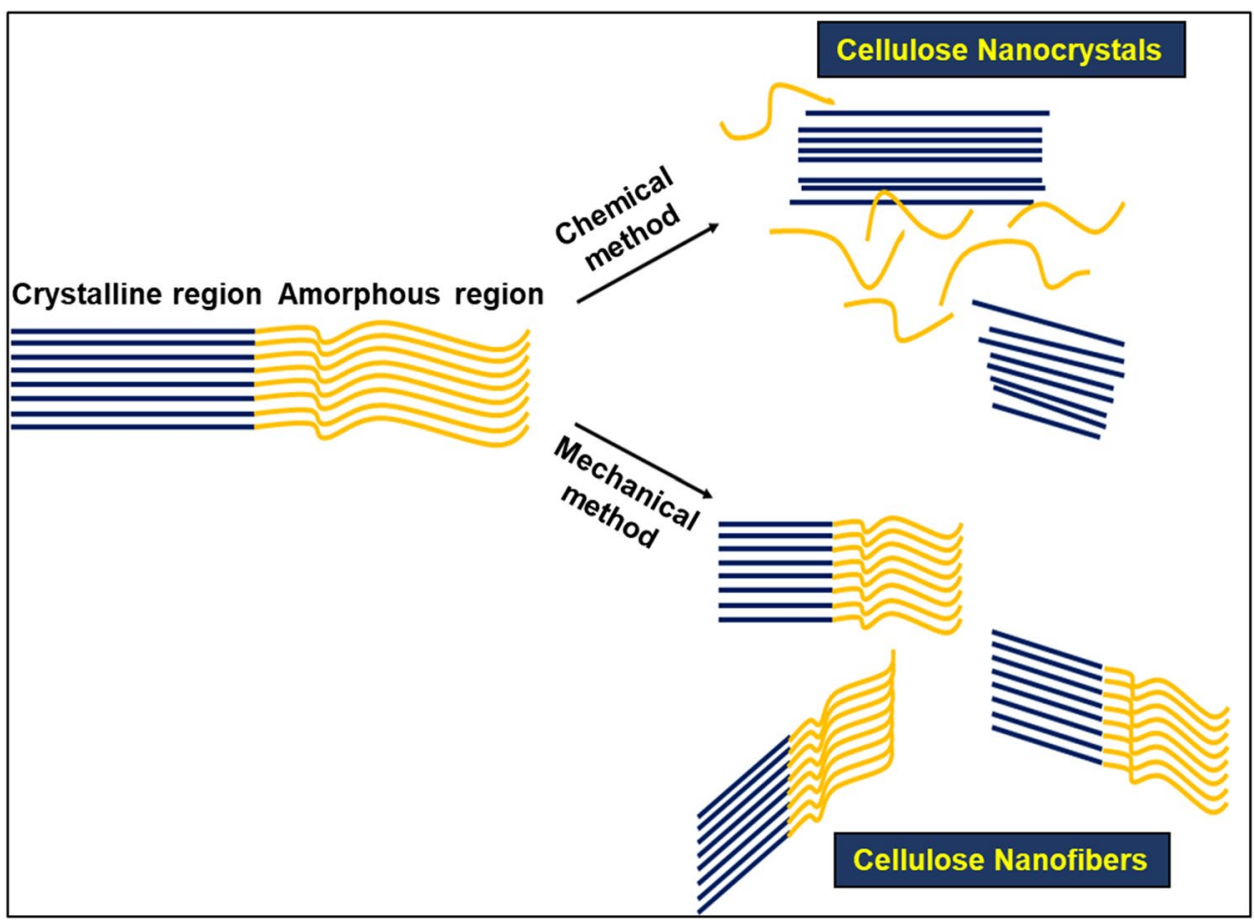


and diameter of $48.1 \pm 14.6 \mathrm{~nm}$ ( $\mathrm{Lu}$ and Hsieh 2012). Neto et al. (2013) hydrolyzed soy hulls at $40{ }^{\circ} \mathrm{C}$ and analyzed the aggregates of nanocrystals and individual needle-like particles. After 30 min of treatment, nanocellulose of 73.5\% crystallinity with an average cross-sectional dimension of $2.73 \mathrm{~nm}$ was obtained. Conversely, with the increase in time, a decrease in crystallinity was observed, which was attributed to the removal of both amorphous and crystalline domains.

Teixeria et al. (Teixeira et al. 2009) isolated cellulose nanofibers (length: 360-1799 nm and diameter: 2-11 nm) from cassava bagasse and reported the $54.1 \%$ crystallinity after acidic treatment. By an alkali treatment at $130{ }^{\circ} \mathrm{C}$, followed by sulfuric acid hydrolysis, cellulose whiskers of diameter ranging from 20 to $40 \mathrm{~nm}$ with the crystallinity of $73.4 \%$ were prepared from branch-barks of white mulberry. Ribbon-like cellulose nanocrystals ( $33 \%$ weight by weight) were extracted from miscanthus $\mathrm{x}$ giganteus pulp by hydrochloric acid and sulfuric hydrolysis with the crystallinity index of $94 \%$; $60-70$ aspect ratio; width $8.5 \mathrm{~nm}$ and the thickness of $2.8 \mathrm{~nm}$ (Cudjoe et al. 2017).

Alternatively, nanocellulose can be prepared by the oxidative process. Before mechanical treatment, cellulose may be oxidized with 2,2,6,6-tetramethylpiperidine-1-oxyl, which is known as TEMPO, radicals under mild aqueous conditions, in which hydroxyl groups of cellulose converted to a carboxylic acid (Isogai et al. 2011; Habibi and Vignon 2008). Saito et al. (2009) pretreated hardwood with the 2,2,6,6-tetramethylpiperidine-1-oxyl-mediated and extracted cellulose in the range of 3-4 nm width. Recently, Patiño-Masó et al. (2019) prepared cellulose nanofibers using oxidation with the same reagent (2,2,6,6-tetramethylpiperidine-1-oxyl) on bleached kraft eucalyptus pulp. Some researchers reported a similar study yielded cellulose nanofibers by the same oxidation approach with the same reagent (Petroudy et al. 2018; Iwamoto et al. 2011).

The utilization of enzymes, which is known to be an environmentally friendly treatment process, has also been reported. Henriksson et al. (2007) and Paakko et al. (2007) disintegrated wood fiber pulp into the microfibrillated cellulose by using endoglucanase. Tibolla et al. (2014) compared the enzymatic pretreatment with chemical pretreatment for the preparation of cellulose nanofibers. Zhao et al. (2017) extracted cellulose nanofibers from the softwood and hardwood with endoglucanase. On the whole, chemically treated biomass yield is much higher than that of enzymatically produced nanocellulose; however, the enzymatic approach is more favorable to maintain the principles of green chemistry.

In recent times, the production of cellulose nanoparticles at a commercial scale is also reported. Inventia, Sweden, set up the first pilot-scale production plant in 2011. Conversely, most of the cellulose nanoparticles' plants are based in Europe, Canada, the USA, China, Japan, Iran, and India.
Among countless methods for the production of cellulose nanoparticles, acid hydrolysis for cellulose nanocrystals and mechanical processes for the cellulose nanofibers are the most trusted methods. Although these two methods considered successful at the industrial scale, the high cost of chemicals, maintenance of equipment operated in the acidic environment, acidic effluent, and energy-intensive mechanical processes are the major concerns associated with these two methods (Sharma et al. 2019).

\section{Properties of cellulose nanoparticles}

On decreasing the dimensions of cellulose from microscale to nanoscale, quite a few properties may alter that are expected to open new pathways for the utilization of nanoparticles. Some of the major properties that can be influenced are specific surface area, aspect ratio, morphological, tensile, rheological, and thermal properties.

\section{Specific surface area}

Reduction in the particle size leads to an increase in specific surface area (total surface area per unit mass). Cellulose nanoparticles, especially cellulose nanocrystals, have a large surface area which is ascribed to the nanodimension of the structural elements, which are greater than $100 \mathrm{~m}^{2} \mathrm{~g}^{-1}$ to several hundreds of $\mathrm{m}^{2} \mathrm{~g}^{-1}$. The large surface area of cellulose nanoparticles governs the interaction (hydrogen bonding) between the filler and the polymer matrix, as the high aspect ratio diameter of cellulose nanoparticles offer improved surface contact of cellulose with the respective polymer. The superior surface contact enhances the surface reactivity and chain mobility of the polymer, consequently improving the properties of the nanocomposites. The average geometrical dimensions method is considered more reliable for the determination of specific surface area compared to the gas adsorption isotherm technique, which is often incorrect because of the aggregation of particles upon drying. While converting sisal fibers-extracted cellulose nanofibers to cellulose nanocrystals, the values of $51 \mathrm{~m}^{2} \mathrm{~g}^{-1}$ and $533 \mathrm{~m}^{2} \mathrm{~g}^{-1}$, respectively, were reported (Siqueira et al. 2009). A sharp upsurge is noted when the diameter falls below $20 \mathrm{~nm}$, which corresponds to the diameter range of cellulose nanocrystals (Dufresne 2019). Besides the high surface area, cellulose nanoparticles tend to agglomerate within themselves, resulting in poor composite reinforcement.

\section{Aspect ratio}

Aspect ratio, the ratio of the length to width, is deliberated as an important parameter for the cellulose nanoparticles, as the aspect ratio signifies the reinforcing properties and 
the anisotropic phase formation. It is well known that the mechanical percolation approach is extremely pertinent to express the mechanical properties of cellulose-based nanocomposites when prepared through casting or evaporation techniques. This method proposes the formation of a continuous network of nanoparticles, combined through hydrogen bonding, which leads to an exceptional strengthening effect. Additionally, this can only occur above a critical volume fraction of filler, which is defined as the percolation threshold. It was experiential that the tensile modulus amplifies on increasing the aspect ratio of reinforcing nanoparticles. The determination of the aspect ratio of cellulose nanocrystals is easier, and it is found to depend on the source of cellulose and the conditions under which hydrolysis had occurred. For instance, the aspect ratio of cotton, tunicin, and soy hulls are 10, 67, and 100, respectively (Dufresne 2017a, 2019). A higher aspect ratio could improve the properties of nanocomposites due to its nanoenabled functional features, such as percolation and tangling effects.

\section{Morphology}

Dimensions and shapes of cellulose nanoparticles have significant importance, for both research and practical applications. Many properties of cellulose nanoparticles and cellulose nanoparticles-based materials are stringently influenced by the characteristic dimensions and shapes of nanoparticles. Without any clear idea of nanoparticles arrangements, the end properties of the composites cannot be predicted. For example, in Isogai's review (2011), it is recommended that the cellulose nanoparticles can be used as reinforcement in bulk materials, cellulose nanoparticles can be used as independent materials or as filler, 2,2,6,6-tetramethylpiperidine1-oxyl-oxidized or known as TEMPO-oxidized cellulose fibers and nanofibrils can be used as a coating, and possibly also as a filler. Li et al. (2013) reported that cellulose nanocrystals extracted from cotton linters could improve the performance of packaging films if used as a coating. Dimensions and crystallinity of cellulose nanoparticles (cellulose nanocrystals and cellulose nanofibers) extracted from various renewable sources are listed in Tables 2 and 3.

In the following tables, dimensions and crystallinity were discussed according to the different sources from which cellulose nanoparticles were extracted. The vast distribution of sources provides assistance to choose the raw material based on the cost, availability, and potential applications. Second, in the tables, dimensions of the cellulose nanoparticles are listed; these dimensions clearly show that the diameter varies in the range of 3 to $50 \mathrm{~nm}$. The length range of cellulose nanoparticles is wide, which is likely to be dependent on the different conditions of acidic hydrolysis treatment. The aspect ratio of tunicate, ramie, algae, banana, coconut husk, and bacterial cellulose nanocrystals are relatively larger than the rest of the others, pointing out that cellulose nanocrystals have inherently exceptional mechanical properties.

Microfibrillated cellulose, on the other hand, exhibits a larger diameter, which is attributed to the limitations of mechanical treatments. Microfibrillated cellulose is significantly longer than cellulose nanoparticles, resulting in a higher aspect ratio and more suitable as a promising filler.

\section{Mechanical properties}

Mechanical properties of fillers play a substantial role in determining the absolute properties of a composite. It is also known to be helpful in the selection of materials for reinforcement purposes. In cellulose nanoparticles, the crystalline region delivers high stiffness and good strength to the polymer matrix. Savadekar and Mhaske (2012) reported that cellulose nanocrystals displayed high elastic modulus (up to $145 \mathrm{GPa}$ ) and Young modulus (150 GPa). The Young axial modulus and mechanical strength of cellulose nanocrystals are estimated to be five times higher than magnesium alloy or mild steel. Every kind of cellulose has its own crystalline structure, which ultimately results in different mechanical properties, as mechanical properties depend on the crystallinity of cellulose. A wide range of values for tensile modulus (56-220 GPa), with an average of $130 \mathrm{GPa}$, have been reported (Dufresne 2017b). Lower crystallinity of cellulose nanofibers results in a lower modulus of around $100 \mathrm{GPa}$ (average value) (Dufresne 2013). Furthermore, cellulose with high crystallinity and a density of around $1.5-1.6 \mathrm{~g} \mathrm{~cm}^{-3}$ can be considered as a lightweight material.

Specific tensile modulus, the ratio between tensile modulus and density, for cellulose nanocrystals and cellulose nanofibers was about 85 and $65 \mathrm{~J} / \mathrm{g}$, respectively, while for steel, it is approximately $25 \mathrm{~J} \mathrm{~g}^{-1}$ (Dufresne 2017b). According to Chen et al. (2011b), cellulose nanoparticles can achieve higher properties for nanocomposites which are attributed to its fibrous structure, which can be adjusted and aligned along a matrix axis. The reason behind the recent use of cellulose in composites formation is its high specific modulus, strength, and low energy consumption compared to other conventional materials. Mohamed et al. (2015) observed that the reinforcement of cellulose nanocrystals into polylactic acid positively affected the thermal and mechanical properties of nanocomposites.

\section{Thermal properties}

The cellulosic-based materials are characterized by their quick thermal deterioration at the low to moderate temperature ranges that occur at about $400{ }^{\circ} \mathrm{C}$ and below. The high crystalline structure of cellulose is the chief factor of the high thermal resistance. Due to the strong stabilization of the hydrogen bond, cellulose chains are packed in a 
Table 2 Morphological properties of cellulose nanocrystals, including length, width, and crystallinity, along with the characterization techniques used for the identification of morphology (Li et al. 2015a)

\begin{tabular}{|c|c|c|c|c|c|}
\hline Type & Extraction method & Length (nanometer) & Width (nanometer) & Crystallinity (\%) & $\begin{array}{l}\text { Characterization for morphol- } \\
\text { ogy }\end{array}$ \\
\hline Aivcel & Sulfuric acid & $105 \pm 35$ & 15 & - & $\begin{array}{l}\text { Transmission electron micros- } \\
\text { copy }\end{array}$ \\
\hline Aivcel & Sulfuric acid & $128 \pm 4$ & $5.5 \pm 0.1$ & 83 & $\begin{array}{l}\text { Transmission electron micros- } \\
\text { copy }\end{array}$ \\
\hline Algae (Valonia) & $\begin{array}{l}\text { Hydrochloric acid + enzy- } \\
\text { matic }\end{array}$ & $>1000$ & $10-20$ & - & $\begin{array}{l}\text { Transmission electron micros- } \\
\text { copy }\end{array}$ \\
\hline Bamboo & Sulfuric acid & $200-500$ & $<20$ & 72 & $\begin{array}{l}\text { Transmission electron micros- } \\
\text { copy }\end{array}$ \\
\hline Bamboo & Sulfuric acid & $300-400$ & $5-14$ & - & Atomic force microscopy \\
\hline Bamboo & Sulfuric acid & $\sim 500$ & $4-14$ & - & Atomic force microscopy \\
\hline Bagasse & Sulfuric acid & $96.7 \pm 39$ & $7.5 \pm 2.3$ & 89 & $\begin{array}{l}\text { Transmission electron micros- } \\
\text { copy }\end{array}$ \\
\hline Bagasse & Sulfuric acid & 170 & 35 & 85 & $\begin{array}{l}\text { Transmission electron micros- } \\
\text { copy }\end{array}$ \\
\hline Bagasse & $\begin{array}{l}\text { Sulfuric acid + alkaline treat- } \\
\text { ment }\end{array}$ & $>1000$ & $20-25$ & - & Scanning electron microscopy \\
\hline Banana & Sulfuric acid & $500-1000$ & 20 & - & $\begin{array}{l}\text { Transmission electron micros- } \\
\text { copy }\end{array}$ \\
\hline Banana & Fermentation & $>1000$ & 50 & 71 & Scanning electron microscopy \\
\hline Banana & Hydrochloric acid & $624-855$ & $16-17$ & - & $\begin{array}{l}\text { Transmission electron micros- } \\
\text { copy }\end{array}$ \\
\hline Bacterial cellulose & Hydrochloric acid & $290 \pm 130$ & $20 \pm 5$ & $91-96$ & $\begin{array}{l}\text { Transmission electron micros- } \\
\text { copy }\end{array}$ \\
\hline Bacterial cellulose & Hydrochloric acid & $88 \pm 5$ & $6.5 \pm 0.2$ & 70 & $\begin{array}{l}\text { Transmission electron micros- } \\
\text { copy }\end{array}$ \\
\hline Buckeye & $\begin{array}{l}\text { Sulfuric acid } \\
\text { Hydrochloric acid }\end{array}$ & $60-576$ & $60-576$ & $67-82$ & $\begin{array}{l}\text { Transmission electron micros- } \\
\text { copy, Scanning electron } \\
\text { microscopy }\end{array}$ \\
\hline Cassava & Sulfuric acid & 1150 & 15 & 54.1 & $\begin{array}{l}\text { Transmission electron micros- } \\
\text { copy }\end{array}$ \\
\hline Curaua & Sulfuric acid & 185 & 11 & $83-85$ & $\begin{array}{l}\text { Transmission electron micros- } \\
\text { copy }\end{array}$ \\
\hline Coconut husk & Sulfuric acid & $>1000$ & 2.3 & $50-57$ & Atomic force microscopy \\
\hline Eucalyptus & Sulfuric acid & $142 \pm 49$ & $11 \pm 4$ & 82 & $\begin{array}{l}\text { Transmission electron micros- } \\
\text { copy }\end{array}$ \\
\hline Flax & Ultra-sonication & $>1000$ & $15-100$ & 78.3 & Scanning electron microscopy \\
\hline Flax & Hydrochloric acid & $144 \pm 5$ & $38 \pm 0.1$ & 75 & $\begin{array}{l}\text { Atomic force microscopy, } \\
\text { Transmission electron } \\
\text { microscopy }\end{array}$ \\
\hline Grape & Sulfuric acid & Sphere & 48.1 & 55 & $\begin{array}{l}\text { Transmission electron micros- } \\
\text { copy }\end{array}$ \\
\hline Hemp & Sulfuric acid & $160 \pm 72$ & $5 \pm 1$ & - & Atomic force microscopy \\
\hline Hemp & Sulfuric acid & $144 \pm 41$ & 3 & - & $\begin{array}{l}\text { Transmission electron micros- } \\
\text { copy }\end{array}$ \\
\hline Hemp & Hydrochloric acid & $148 \pm 3$ & $5.8 \pm 0.1$ & 73 & $\begin{array}{l}\text { Transmission electron micros- } \\
\text { copy }\end{array}$ \\
\hline Hardwood & Sulfuric acid & $518 \pm 183$ & $22 \pm 13$ & - & $\begin{array}{l}\text { Transmission electron micros- } \\
\text { copy }\end{array}$ \\
\hline Jute & $\begin{array}{l}\text { 2,2,6,6-Tetramethylpiperi- } \\
\text { dine-1-oxyl }\end{array}$ & $100-200$ & $3-10$ & 69.7 & $\begin{array}{l}\text { Transmission electron micros- } \\
\text { copy }\end{array}$ \\
\hline Kenaf Bast & Sulfuric acid & $100-20$ & $10-20$ & $75-82$ & $\begin{array}{l}\text { Transmission electron micros- } \\
\text { copy }\end{array}$ \\
\hline
\end{tabular}


Table 2 (continued)

\begin{tabular}{|c|c|c|c|c|c|}
\hline Type & Extraction method & Length (nanometer) & Width (nanometer) & Crystallinity (\%) & $\begin{array}{l}\text { Characterization for morphol- } \\
\text { ogy }\end{array}$ \\
\hline Mengkuang & $\begin{array}{l}\text { Alkali treatment }+ \text { sulfuric } \\
\text { acid }\end{array}$ & $50-400$ & $5-25$ & 70 & $\begin{array}{l}\text { Transmission electron micros- } \\
\text { copy }\end{array}$ \\
\hline Mulberry & $\begin{array}{l}\text { Alkali treatment }+ \text { sulfuric } \\
\text { acid }\end{array}$ & $400-500$ & $20-40$ & 73.4 & Atomic force microscopy \\
\hline Pea Hull & Sulfuric acid & $400 \pm 200$ & $12 \pm 6$ & - & $\begin{array}{l}\text { Transmission electron micros- } \\
\text { copy }\end{array}$ \\
\hline Ramie & Sulfuric acid & $50-250$ & $3-15$ & 87.8 & Atomic force microscopy \\
\hline Ramie & Sulfuric acid & $120-280$ & $6-8$ & - & $\begin{array}{l}\text { Transmission electron micros- } \\
\text { copy }\end{array}$ \\
\hline Ramie & Sulfuric acid & $100-250$ & $3-10$ & - & $\begin{array}{l}\text { Transmission electron micros- } \\
\text { copy }\end{array}$ \\
\hline Ramie & Sulfuric acid & $75-193$ & $6.3-15.3$ & - & $\begin{array}{l}\text { Transmission electron micros- } \\
\text { copy }\end{array}$ \\
\hline Ramie & Sulfuric acid & $100-150$ & $6-8$ & - & $\begin{array}{l}\text { Transmission electron micros- } \\
\text { copy }\end{array}$ \\
\hline Sisal & Sulfuric acid & 195 & 15 & - & $\begin{array}{l}\text { Transmission electron micros- } \\
\text { copy }\end{array}$ \\
\hline Sisal & Sulfuric acid & $120-370$ & $5-8$ & - & $\begin{array}{l}\text { Transmission electron micros- } \\
\text { copy }\end{array}$ \\
\hline Sisal & $\begin{array}{l}\text { Enzymatic + shearing + sul- } \\
\text { furic acid }\end{array}$ & $170-300$ & $6-8$ & - & $\begin{array}{l}\text { Transmission electron micros- } \\
\text { copy }\end{array}$ \\
\hline Sisal & Enzyme & $230-560$ & $6-13$ & - & $\begin{array}{l}\text { Transmission electron micros- } \\
\text { copy }\end{array}$ \\
\hline Sisal & Sulfuric acid & $215 \pm 67$ & $5 \pm 1.5$ & - & Scanning electron microscopy \\
\hline Softwood & Sulfuric acid & $234 \pm 60$ & $6 \pm 2$ & - & Atomic force microscopy \\
\hline Softwood & Sulfuric acid & 2500 & 25 & - & $\begin{array}{l}\text { Transmission electron micros- } \\
\text { copy }\end{array}$ \\
\hline Tunicate & Sulfuric acid & 1000 & 15 & - & $\begin{array}{l}\text { Transmission electron micros- } \\
\text { copy }\end{array}$ \\
\hline Tunicate & Sulfuric acid & $500-2000$ & 15 & - & $\begin{array}{l}\text { Transmission electron micros- } \\
\text { copy }\end{array}$ \\
\hline
\end{tabular}

highly ordered manner with a compact structure. The formation of the highly crystalline cellulose is considered to generate high thermal resistance due to a compact crystalline structure which cannot be burned or destroyed by high temperature, averting the cellulose from melting. After the drying of cellulose nanoparticles, a film is formed, which has a low thermal expansion coefficient owing to the high crystallinity and strong cellulose network interactions (Kargarzadeh et al. 2017). While using the low thermal expansion coefficient of cellulose nanoparticles, the thermal expansion coefficient of the polymer nanocomposites can be reduced if the strength of the network formed between the cellulose and the polymer is strong. Sulfuric acid hydrolysis significantly reduces the thermal stability of cellulose nanocrystals compared to raw cellulose fibers. It is due to the presence of sulfate ester groups on the surface of the cellulose nanoparticles (Lin and Dufresne 2014).

\section{Rheological properties}

The rheological parameters of cellulose nanocrystals are predominantly influenced by liquid crystallinity, ordering, and gelation properties. The suspensions of dilute cellulose nanocrystals exhibit shear-thinning behavior, which shows concentration dependence at low rates. At higher concentrations, cellulose nanocrystals suspensions are lyotropic, which reveals anomalous behavior which was attributed to the rodshaped nanocrystals that tend to align at a critical shear rate. As the shear rate reaches a critical point, the chirality of the cellulose nanocrystals suspension breaks down in favor of a simple nematic structure (Azizi Samir et al. 2004). Moreover, the aspect ratio of cellulose nanocrystals governs the relaxation time constant, as cellulose nanocrystals with higher aspect ratios stay aligned for longer times even after shear. Besides these, the acid used for the hydrolysis process has an impact on the rheological properties of cellulose nanocrystals 
Table 3 Morphological properties of microfibrillated cellulose, including length, width, and crystallinity, along with the characterization techniques used for the identification of morphology ( $\mathrm{Li}$ et al. 2015a)

\begin{tabular}{|c|c|c|c|c|c|}
\hline Type & Extraction method & Length (nanometer) & Width (nanometer) & Crystallinity $(\%)$ & Characterization for morphology \\
\hline Bagasse & Homogenizer & $>1000$ & $5-18$ & - & Atomic force microscopy \\
\hline Bagasse & Homogenizer & $>1000$ & $6-22$ & - & Atomic force microscopy \\
\hline Bagasse & Homogenizer & $>1000$ & $7-30$ & $74-76$ & Transmission electron microscopy \\
\hline Bamboo & Homogenizer & $>1000$ & $23-42$ & - & Atomic force microscopy \\
\hline Bamboo & Ultra-sonication & $>1000$ & $10-40$ & 55 & Scanning electron microscopy \\
\hline Flax & $\mathrm{HCl}+$ shear homogenizer & $>1000$ & $30-50$ & 80 & Transmission electron microscopy \\
\hline Flax & Ultra-sonication & $>1000$ & $15-100$ & 78.3 & Scanning electron microscopy \\
\hline Hardwood & Grinder & $200-3000$ & $10-20$ & - & Atomic force microscopy \\
\hline Perennial Ryegrass & Homogenizer & $300-4600$ & $3-9.1$ & - & Transmission electron microscopy \\
\hline Rice Straw & Homogenizer & $>1000$ & $2-5$ & 40 & Transmission electron microscopy \\
\hline Rice Straw & Homogenizer & $>1000$ & $4-13$ & - & Transmission electron microscopy \\
\hline Rice Straw & Homogenizer & $>1000$ & $52 \pm 15$ & - & Transmission electron microscopy \\
\hline Sisal & Homogenizer & $>1000$ & $52 \pm 15$ & - & Scanning electron microscopy \\
\hline Sisal & Refining (beater) & $>1000$ & $25-250$ & 87.2 & Scanning electron microscopy \\
\hline Softwood & Ultra-sonication & $>1000$ & $10-20$ & 56 & Scanning electron microscopy \\
\hline Softwood & Homogenizer & $>1000$ & $50-60$ & - & Atomic force microscopy \\
\hline Wheat Straw & Ultra-sonication & $>1000$ & $15-35$ & 59.9 & Scanning electron microscopy \\
\hline Wheat Straw & Homogenizer & $>1000$ & 4 & 80 & $\begin{array}{l}\text { Atomic force microscopy, Trans- } \\
\text { mission electron microscopy, } \\
\text { X-ray diffraction }\end{array}$ \\
\hline
\end{tabular}

suspensions. Crystals treated with sulfuric acid show some shear thinning that is independent of time. In contrast, crystals treated with hydrochloric acid reveal much higher shear thinning behavior, anti-thixotropy at lower concentrations, and thixotropy at higher concentrations (Araki et al. 1999).

Rheometry measurements have been performed on cellulose nanofibers prepared via 2,2,6,6-tetramethylpiperidine1 -oxyl oxidation. These cellulose nanofibers' suspensions also showed shear-thinning behavior following a power-law and thixotropy, which are explained through percolation in the fibrils and flock formation. In this case, gelation was observed to onset (storage modulus four-loss modulus) at about $0.5 \%$ cellulose (Lasseuguette et al. 2008). Li et al. (2015b) investigated the structure-morphology-rheology relationships for cellulose nanoparticles and observed that the viscosity of the cellulose nanocrystals suspensions gradually increases as the concentration increases because of the growth in the collision possibility of cellulose nanocrystals. However, the viscosity monotonically declines as the shear rate increases over the whole investigated shear rate ranges, exhibiting a typical shear thinning behavior.

\section{Preparation of the cellulosic nanocomposites}

Renewability, biodegradability, abundance, and low cost are some of the main highlights of cellulose-containing materials, which suggests that these particles have the potential for a wide range of applications. The most efficient way to exploit and enhance the properties of cellulose nanoparticles is to reinforce it in a polymer. Numerous methods have been discussed in the literature for the preparation of cellulose nanoparticles-based polymer composites. This review aims to present the most promising techniques that are used for the preparation of nanocomposites. Table 4 shows the processing techniques of nanocellulosic based polymer composites extracted from different types of crops. The majority of the cellulose nanocrystals-based polyvinyl alcohol composites are reported to be prepared through solvent casting technique, while solution casting is found to be a more universal or efficient technique that can deal with different kinds of matrices. 
Table 4 Processing techniques for the preparation of cellulose nanoparticles-based polymer composites that are used in the food packaging application

\begin{tabular}{|c|c|c|c|c|c|}
\hline Fibers & Cellulose type & Filler (wt\%) & Matrix & Processing technique & References \\
\hline Barley straw and husk & Cellulose nanocrystals & $1-3$ & - & Solvent casting & Fortunati et al. (2016) \\
\hline Industrial waste cotton & Cellulose nanocrystals & 5 & Polyvinyl alcohol & Solvent casting & $\begin{array}{l}\text { Thambiraj and } \\
\text { Shankaran (2017) }\end{array}$ \\
\hline Sugarcane bagasse & Cellulose nanocrystals & $0.5-10$ & - & Solvent casting & El Achaby et al. (2017) \\
\hline Sugarcane bagasse & Cellulose nanocrystals & $0.5-5$ & - & Solvent casting & El Miri et al. (2015) \\
\hline Kiwi pruning stalk & Cellulose nanocrystals & 3 & - & Solvent casting & Luzi et al. (2017) \\
\hline Softwood kraft pulp & Cellulose nanocrystals & 5 & - & Solvent casting & Khan et al. (2012) \\
\hline Rice straw & Cellulose nanocrystals & $1-5$ & - & Solvent casting & Perumal et al. (2018) \\
\hline Red algae & Cellulose nanocrystals & $1-8$ & Polyvinyl alcohol & Solvent casting & El Achaby et al. (2018) \\
\hline Softwood kraft pulp & Cellulose nanocrystals & $1-8$ & Alginate biopolymer & Solution casting & Huq et al. (2012) \\
\hline Cotton wool & Cellulose nanocrystals & $5-15$ & Polyvinyl alcohol & Solution casting & Popescu (2017) \\
\hline Kenaf fibers & Cellulose nanocrystals & $2-10$ & $\begin{array}{l}\text { Thermoplastic cassava } \\
\text { starch }\end{array}$ & Solution casting & Zainuddin et al. (2013) \\
\hline Wheat straw & Cellulose nanofibers & - & Thermoplastic starch & Solution casting & $\begin{array}{l}\text { Alemdar and Sain } \\
\text { (2008) }\end{array}$ \\
\hline- & Nanocellulose & $0.1-1$ & - & Solution casting & Khan et al. (2010) \\
\hline $\begin{array}{l}\text { Microcrystalline cel- } \\
\text { lulose }\end{array}$ & - & $1-10$ & Alginate biopolymer & Solution casting & Abdollahi et al. (2013) \\
\hline $\begin{array}{l}\text { Bleached dry eucalyp- } \\
\text { tus pulp }\end{array}$ & $\begin{array}{l}\text { Cellulose nanofib- } \\
\text { ers and cellulose } \\
\text { nanocrystals }\end{array}$ & 10 & Polyethylene oxide & Solution casting & Xu et al. (2013) \\
\hline $\begin{array}{l}\text { Gluconacetobacter } \\
\text { Xylinum }\end{array}$ & $\begin{array}{l}\text { Bacterial cellulose } \\
\text { nanocrystals }\end{array}$ & 2 and 4 & $\begin{array}{l}\text { Hydroxyl-propyl- } \\
\text { methyl cellulose }\end{array}$ & Solution casting & George et al. (2014) \\
\hline $\begin{array}{l}\text { Gluconacetobacter } \\
\text { Xylinum }\end{array}$ & $\begin{array}{l}\text { Bacterial cellulose } \\
\text { nanocrystals }\end{array}$ & $1-5$ & Gelatin & Solution casting & George (2012) \\
\hline Acetobacter xylinum & $\begin{array}{l}\text { Bacterial cellulose } \\
\text { nanocrystals }\end{array}$ & 4 & Polyvinyl alcohol & Solution casting & George et al. (2011) \\
\hline Potato & Cellulose nanofibers & 3.3 & Starch & Solution casting & Dufresne et al. (2000) \\
\hline Pea hull fibers & Cellulose nanocrystals & 30 & Starch & Solution casting & Chen et al. (2009) \\
\hline Eucalyptus wood pulp & Cellulose nanocrystals & $0-6$ & - & Solution casting & $\begin{array}{l}\text { de Mesquita et al. } \\
\text { (2012) }\end{array}$ \\
\hline Sunflower stalks & $\begin{array}{l}\text { Cellulose nanofib- } \\
\text { ers and cellulose } \\
\text { nanocrystals }\end{array}$ & 0.4 (volume/volume) & Gelatin & Solution casting & Fortunati et al. (2016) \\
\hline $\begin{array}{l}\text { Jute and bacterial } \\
\text { cellulose }\end{array}$ & $\begin{array}{l}\text { Bacterial cellulose } \\
\text { nanocrystals }\end{array}$ & 50 & Starch matrix & Film casting & $\begin{array}{l}\text { Soykeabkaew et al. } \\
\text { (2012) }\end{array}$ \\
\hline Luffa Cylindrica & Cellulose nanocrystals & $0-12$ & Polycaprolactone & Film casting & Follain et al. (2013) \\
\hline Softwood kraft pulp & Cellulose nanocrystals & 5 & Polycaprolactone & Compression molding & Khan et al. (2013) \\
\hline Cotton linter pulp & Cellulose nanocrystals & $0-30$ & Soy protein isolate & Compression molding & Wang et al. (2006) \\
\hline $\begin{array}{l}\text { Needle leaf bleached } \\
\text { kraft pulp }\end{array}$ & Cellulose nanofibers & 10 & Polylactic acid & Compression molding & Iwatake et al. (2008) \\
\hline $\begin{array}{l}\text { Commercial cotton } \\
\text { paper }\end{array}$ & Cellulose nanocrystals & $1-12$ & - & Casting & Pereda et al. (2014) \\
\hline- & Cellulose nanocrystals & $0-20$ & - & Casting & Azeredo et al. (2010) \\
\hline- & Cellulose nanofibers & 50 & - & Casting & Fernandez et al. (2010) \\
\hline- & Cellulose nanofibers & 32 & - & Casting & Wu et al. (2014) \\
\hline Bamboo & Cellulose nanocrystals & 2 & $\begin{array}{l}\text { Poly(3-hydroxybu- } \\
\text { tyrate) }\end{array}$ & Casting & Dhar et al. (2015) \\
\hline Cotton linter & $\begin{array}{l}\text { Cellulose nanowhisk- } \\
\text { ers }\end{array}$ & $0-20$ & - & Evaporation & Li et al. (2009) \\
\hline Kenaf pulp & Cellulose nanofibers & $1-5$ & Polylactic acid & Twin-screw extrusion & Jonoobi et al. (2010) \\
\hline
\end{tabular}


Table 4 (continued)

\begin{tabular}{lllllc}
\hline Fibers & Cellulose type & Filler $(\mathrm{wt} \%)$ & Matrix & Processing technique & References \\
\hline $\begin{array}{l}\text { Phormium Tenax } \\
\text { leaves }\end{array}$ & Cellulose nanocrystals & $1-3$ & Polylactic acid & $\begin{array}{c}\text { Twin-screw micro } \\
\text { extruder }\end{array}$ & $\begin{array}{c}\text { Fortunati et al. (2014a) } \\
\text { and Fortunati et al. } \\
(2014 \mathrm{~b})\end{array}$ \\
- & $\begin{array}{l}\text { Cellulose nanowhisk- } \\
\text { ers }\end{array}$ & - & Polylactic acid & Melt extrusion & Oksman et al. (2006) \\
& $\begin{array}{l}\text { Cellulose nanocrystals } \\
-\end{array}$ & 10 & Polyvinyl alcohol & Heating & Paralikar et al. (2008) \\
Cellulose nanofibers & 2.5 & $\begin{array}{c}\text { Polyvinyl alcohol } \\
\text { polypyrrole }\end{array}$ & In situ polymerization & Bideau et al. (2016) \\
\hline
\end{tabular}

Fig. 5 Representation of the solvent casting method. Polymer composites can be prepared by mixing the natural fibers into the mixture of polymer and solvent. Appropriate dispersion of the natural fibers in the solution, followed by the evaporation of solvent resulted in the natural fiber-reinforced polymer composites
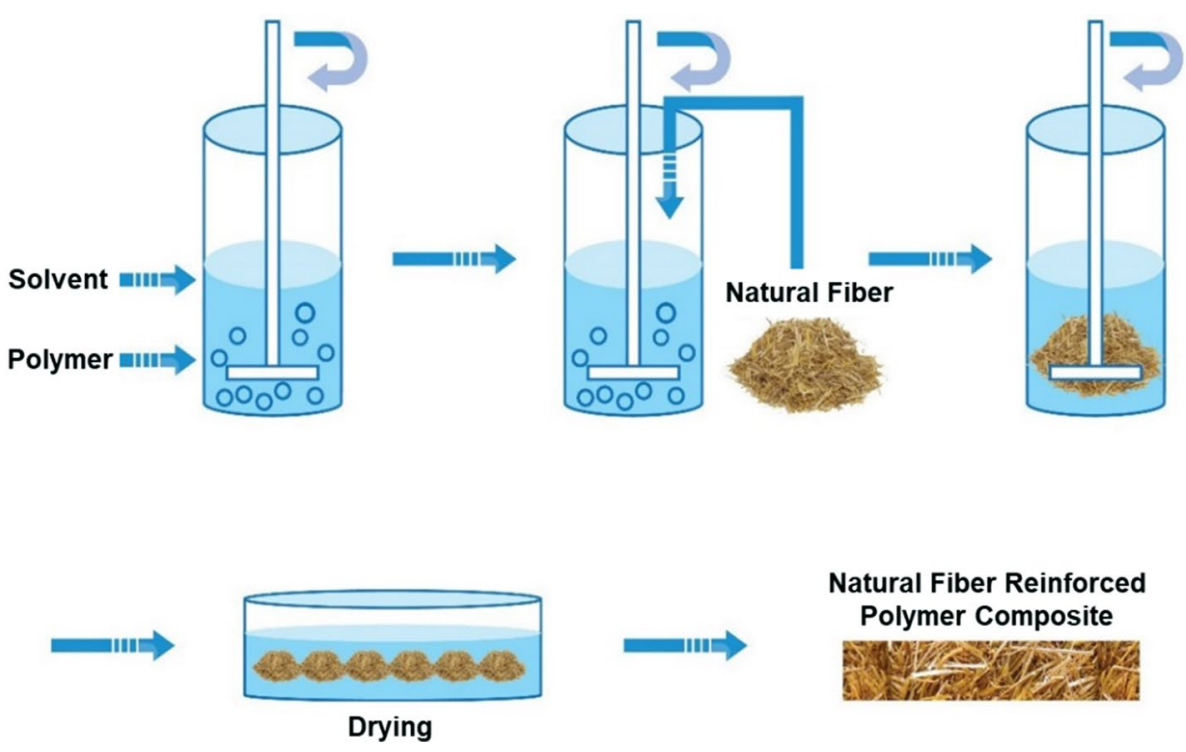

\section{Solvent casting method}

The solvent casting method is known as one of the simplest methods for the preparation of nanocomposites (Fig. 5). Nanocellulose is dispersed within a given medium (water or organic media) to prepare the homogenous suspension at room temperature, followed by the mixing of polymer solutions (Bandyopadhyay-Ghosh et al. 2015). The composites are developed, followed by drying in a vacuum oven. This processing technique strongly influences the mechanical properties of the nanocomposites. It has been reported that the nanocomposites designed by the solution casting technique produce superior results compared to melt extruded composites (Bharimalla et al. 2019). This is attributed to the better dispersion of the nanoparticles and the possibility of hydrogen bonding between the nanofiller and the matrix.

Being an ecological and low-temperature process, the solvent casting technique entails a small quantity of nanofiller to produce the nanocomposite films of uniform thickness. This method has some flaws, which include high time and energy consumption, limited to the laboratory scale, and only useful when a very small amount of filler is required
(Khoshkava 2014). Svagan et al. (2007) efficaciously cast nanostructured cellulose shared with a 50/50 amylopectin-glycerol blend. The resulting film revealed superior tensile strength and modulus owing to the satisfactory dispersion of cellulose. The water uptake property of pure plasticized starch film was reduced in the presence of cellulose nanofibers ( $70 \mathrm{wt} \%$ ). For the production of packaging materials, the casting technique is not a common approach and therefore, very unlikely, it will become the main way to use nanocellulose in packaging applications. It can be considered as a good way to understand, at a laboratory scale, the behavior of cellulose nanoparticles and the opportunities for producing cellulosic nanocomposites using technologies more reliable for larger productions (Bharimalla et al. 2017).

\section{Melt intercalation process}

In 1993, the melt intercalation process was used to prepare polymer composites. Vaia (1993) first used this method in 1993 to process polymer composites. Since then, it is known as the most favorable and valuable technique for the synthesis of nanocomposites. This method is used in the polymer 
Fig. 6 Cellulose nanoparticlesbased polymer composites preparation techniques. a Melt intercalation process for the preparation of composites, in which polymer solubilize and nanocellulose swell in the solvent, respectively. The solubilize polymer and swell nanocellulose combined to form melt-intercalated cellulosic nanocomposites; b In situ polymerization mechanism for the preparation of nanocomposites using monomers and nanocellulose (Bharimalla et al. 2017) (a)

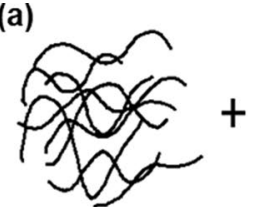

Polymer

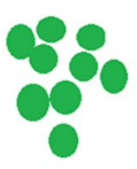

Nanocellulose

(b)

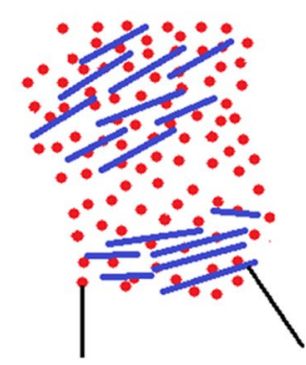

Monomers

\section{In Situ Polymerization}

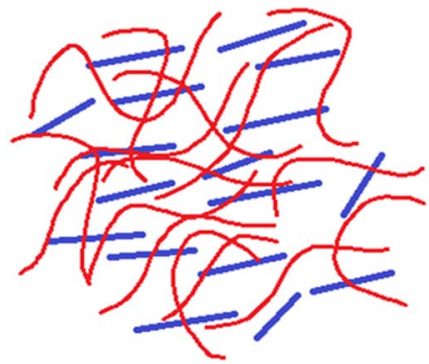

processing industries through compounding devices, such as extruders or mixers. The process of melt intercalation is shown in Fig. 6a. This is a top-down method that involves the mixing of nanoparticles with a fused polymer. Nanocomposites are formed when the mixture of filler and the polymer are above the glass transition temperature. Intercalation is induced when polymer chains penetrate the reinforcement. On compatibility (filler and matrix) terms, either intercalated nanocomposites or exfoliated nanocomposites can be obtained. Eco-friendly and simple melt intercalation process decreases the interfacial tension and improves the matrix reinforcements interactions (Bharimalla et al. 2019). Those polymers which are not suitable for solution intercalation, in situ polymerization or adsorption methods can be used to prepare the nanocomposites. This method is more flexible and does not require chemical reactions or solvents.

\section{In situ polymerization}

In situ polymerization is the first method used for the synthesis of nanocomposites. The process mechanism of the in situ polymerization is shown in Fig. 6b. The in situ polymerization of monomers with nanocellulosic fillers is beneficial compared to the conventional processing approaches because even dispersion of the nanofiller in the polymer can be attained by reducing the agglomeration. Reduction in the moisture absorption behavior and the improvement in the biodegradability of the nanocomposites are achieved by this method. However, this method is more specific because it is only used when polymerization is carried out in the liquid phase, where liquid monomers are polymerized in the presence of cellulose (Bharimalla et al. 2019). Conventionally, this process is used to synthesize thermoplastic nanocomposites. At the same time, for thermosets, a curing agent or peroxide is added to initiate the polymerization or increase in temperature can also help in initiating the polymerization reaction. The in situ polymerization method can be set up at an industrial scale and can significantly increase the development of cellulosic nanocomposites products. Many novel cellulosic nanocomposites have been made through this technique with potential applications, e.g., polymethylmethacrylate, polyacrylamide, polyurethane, polypyrrole (Bharimalla et al. 2019).

\section{Coating}

Coatings lead to a composite structure as thin layers, either external or packed inside two substrates ( $\mathrm{Li}$ et al. 2019). The layer thickness varies from one-tenth of the nanometer to micrometer range. The high performance and functional biomaterials thin layer composites can replace thick oil-based conventional plastics films. To enhance the sustainability of packaging materials, a prodigious interest is developing in the favor of coating technology, which in particular lessens the thickness of oil-based conventional plastic films, by using a thin layer of functional and 
high performing bio-based material. Highly crystalline cellulose nanoparticles were investigated as a functional layer on various substrates. Aulin et al. (2010) coated carboxymethylated microfibrillated cellulose on papers and observed the oxygen permeability at different relative humidity levels. At very low relative humidity level, the microfibrillated cellulose films exhibited considerably low oxygen permeability, like that of conventional synthetic films (such as ethylene-vinyl alcohol). Meanwhile, the dense structure formed because of the cellulose layers and resulted in high oil barrier properties. On the other hand, at higher relative humidity values, an increase in oxygen permeability was observed.

Hult et al. (2010) investigated the coating of the microfibrillated cellulose on the papers and paperboards. Their results showed that the coating technology, as a high barrier, is beneficial in packaging applications. Sanchez-Garcia et al. (2010) enhanced the water barrier properties by incorporating the cellulose nanoparticles in a carrageenan matrix (novel bio-nanocomposites) suggested for applications in food packaging and coating applications (Kuswandi 2017). Da Silva et al. (2012) produced nanocomposite films of cassava starch plasticized with the sucrose and inverted sugar and reinforced by the cellulose nanocrystals extracted from the eucalyptus. Besides good mechanical properties, the water resistance properties of the nanocomposites were meaningfully improved with the addition of cellulose nanocrystals (which formed a rigid network because of the high crystallinity of cellulose nanocrystals). Moreover, their results may be applied to the development of biodegradable packaging to enhance the shelf life of food products. In conclusion, the coating technique has great potential in producing novel materials, especially novel packaging materials (Kuswandi 2017; Ferrer et al. 2017).

\section{Properties of cellulosic nanocomposites}

Composite materials are known to have enhanced capabilities compared to the parent material, as they are reinforced with fillers. Polymer composites based on cellulose nanoparticles exhibit extraordinary mechanical properties even at low loading percentages (Dufresne 2017a). The reason behind such magnificent properties is a network of nanoparticles spread in the polymer matrix. The aspect ratio of cellulose nanoparticles significantly influences the stiffness, as the aspect ratio is directly related to mechanical properties (Bras et al. 2011). With the increase in aspect ratio, the stiffness of the percolating cellulose nanocrystals increases, and thus mechanical properties are increased. Some important properties of cellulose nanoparticles-based polymer composites are represented in Table 5.

\section{Food packaging applications}

Cellulose nanoparticles reinforced polymer composites for the food packaging applications are represented in Table 6. The nanoscale dimensions of the cellulose nanoparticles can build a strong entangled nanoporous network that allows scientists and technologists to use it most efficiently. Plentiful materials have been incorporated with cellulose nanoparticles by various approaches to obtain multifunctional properties, such as enhanced mechanical and barrier properties, improved coloration, and dyeing. Chitin, a second most abundant natural biopolymer, is a non-toxic, biodegradable, biofunctional, and biocompatible material. It has strong antibacterial and antifungal activities, according to many researchers (Darmadji and Izumimoto 1996). Films made from chitin have been used successfully as a packaging material. Moreover, it was revealed that cellulose nanocrystals loading (3-5\%) in chitin gave the best tensile strength values, which is attributed to the formation of a percolating network and stronger filler-matrix interactions.

The surface morphology of the nanocomposites indicated the homogenous structure due to the adequate dispersion of the cellulose nanocrystals into the chitin matrix. Because of the excellent barrier properties and mechanical properties, the film produced would have promising impacts on food packaging (Jo et al. 2001). Elongation decreased, but Young modulus and tensile strength increased at higher concentrations of cellulose in the matrix. Boumil et al. (2013) prepared antimicrobial diffusion films for food applications, which showed the highest tensile strength in storage. Nanocomposite based on nanocellulose and alginate biopolymer was developed by Abdollahi et al. (2013) using solution casting method and found that with the increase in nanocellulose content from 0 to $5 \%$, the tensile strength of the composite increased; however, the additional increment of filler decreased the tensile strength. High (10\%) increment of cellulose nanoparticles in the matrix reduced the transparency of the composite film, which suggested the agglomeration of filler in the matrix results in poor mechanical and structural properties.

Polylactic acid, a versatile biodegradable polymer, is a suitable matrix for the preparation of cellulosic-based nanocomposites. The effect of cellulose nanofibers in the polylactic acid matrix, in terms of mechanical properties and dynamic mechanical properties for food packaging, was investigated by Jonoobi et al. (2010). They observed that the nanocomposite films showed an increase in the tensile strength and Young modulus until 5\% cellulose content. A study conducted by Ghaderi et al. (2014) on the cellulose nanofibers, extracted from the sugarcane 


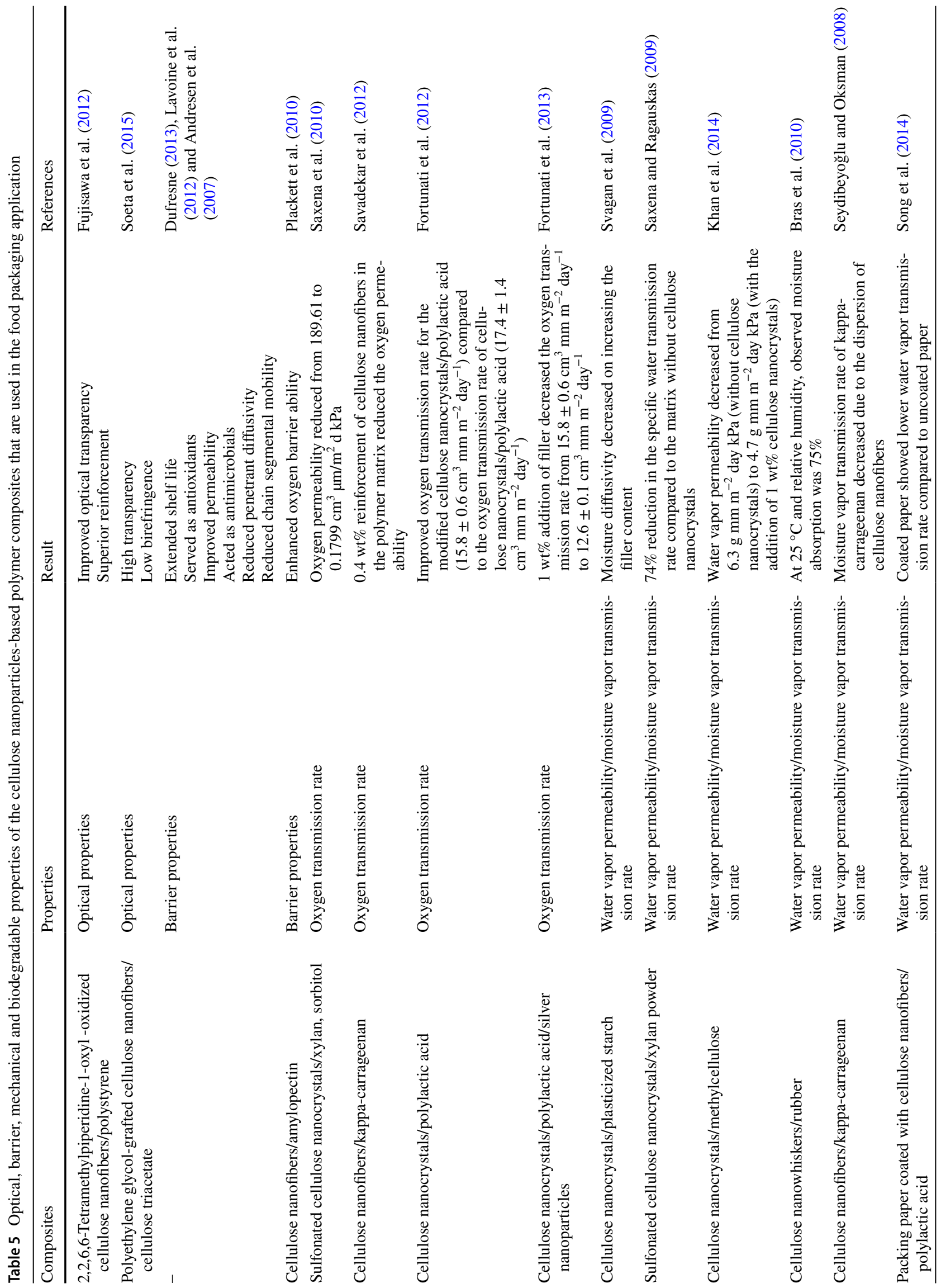




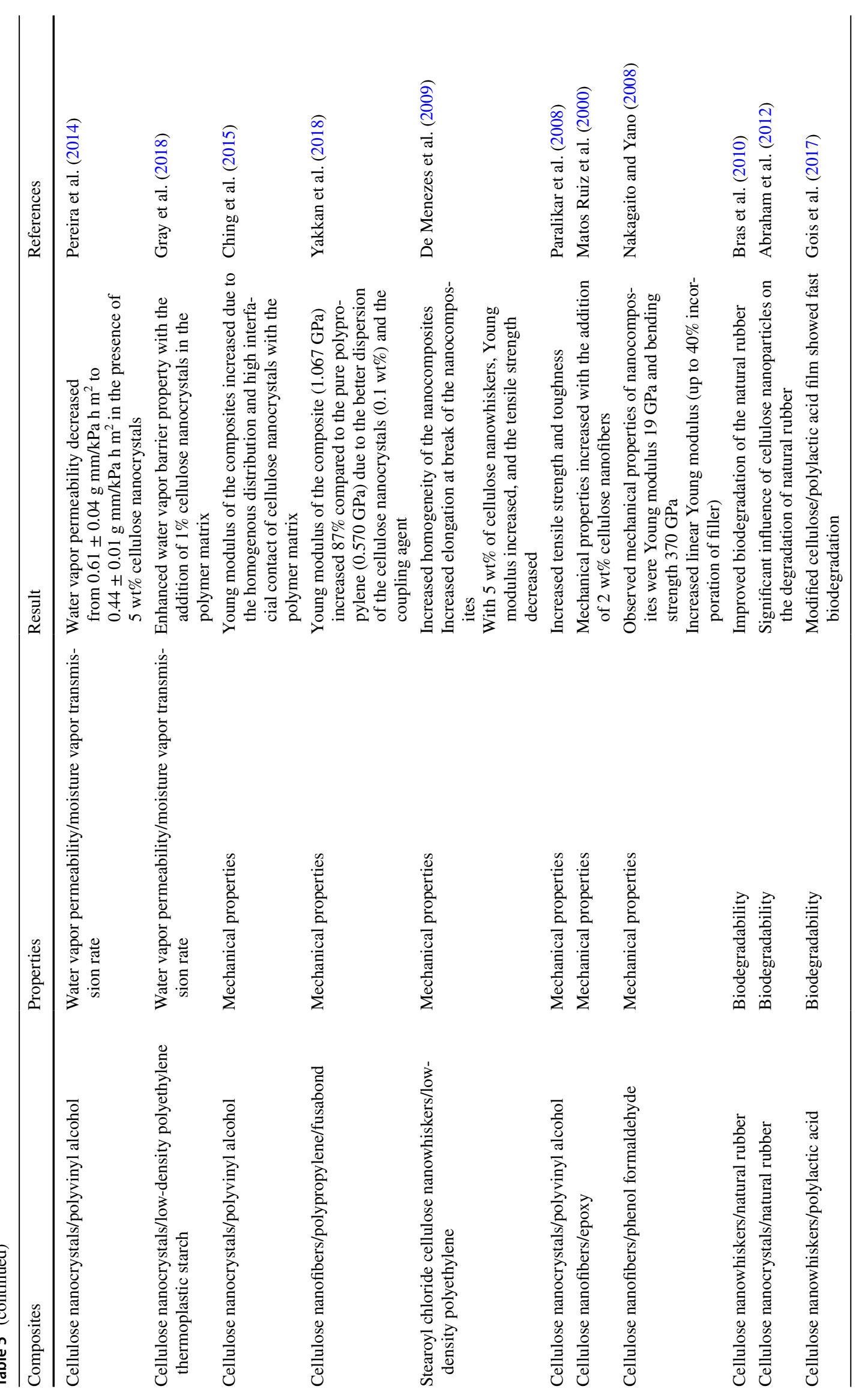




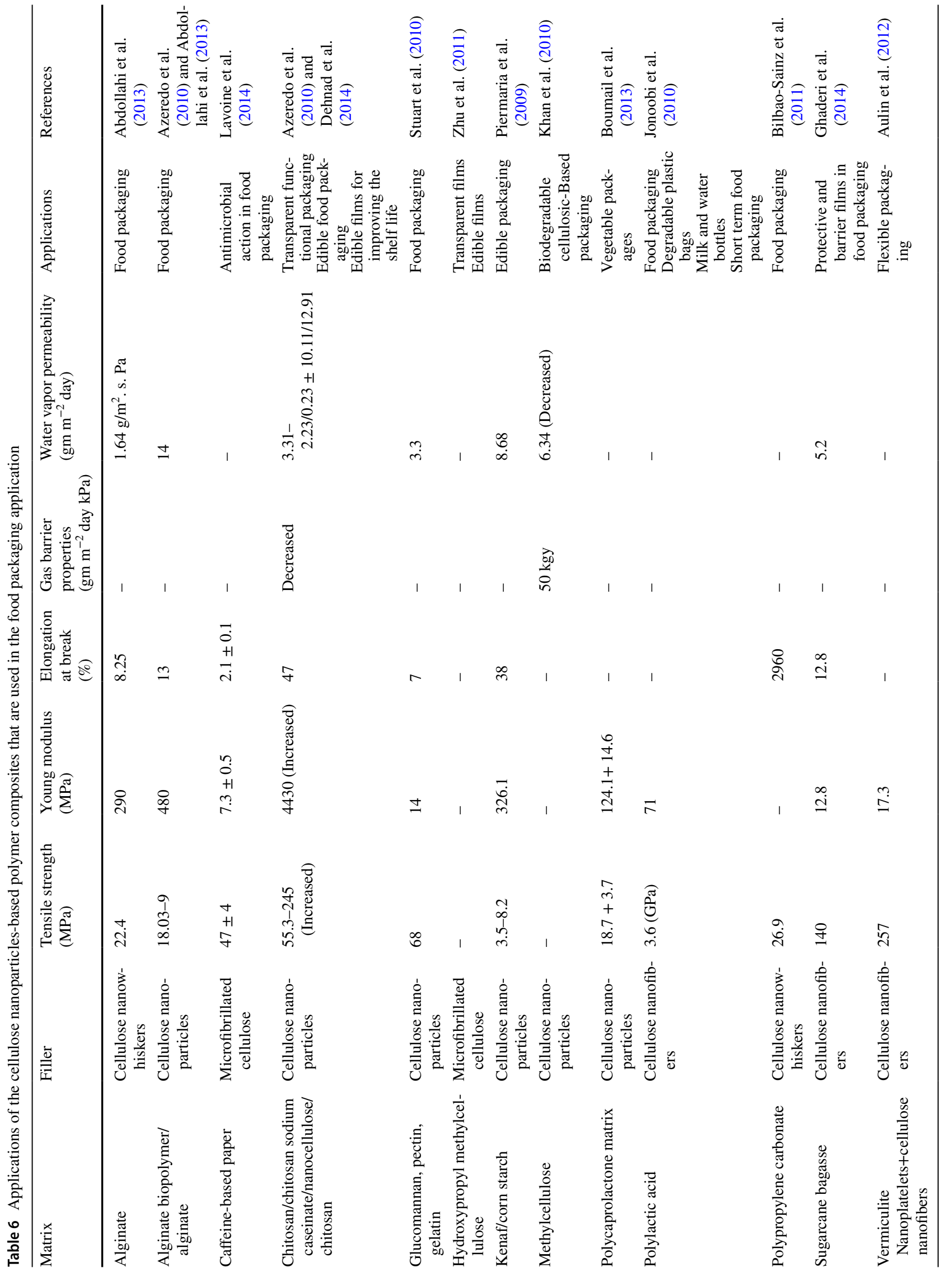


bagasse, showed that water vapor permeability of nanocomposites increased with more dissolution time. The preparation of polylactic acid composites is a method to improve properties. Song et al. (2014) observed that incorporating modified nanocellulose fibers in the polylactic acid improves the water vapor barrier properties of the matrix (Bharimalla et al. 2019). Polylactic acid is a food packaging polymer for short-term products, for instance, drinking cups, containers, wrappers, and laminated films; however, water vapor and gas barrier properties of polylactic acid is not efficient for long term solutions. Besides cellulosic nanocomposites, some of the efficient biocomposites reinforced with inorganic filler are listed in Table 7.

\section{Sustainable packaging}

Sustainable packaging refers to the manufacturing of packaging films by using recyclable and biodegradable materials with their proper life cycle assessment. The proper measure of life cycle assessment helps to eliminate ecological footprints and their negative impact on the environment. The measured use of sustainable packaging materials ensures a cleaner environment for future generations. The organization of sustainable packaging outlines packaging that is: (http://www.sustainablepackaging.org, accessed 10 December 2011)

1. Favorable, harmless, and vigorous for the populations throughout its life cycle.

2. Fulfills market standards for both presentation and price.

3. Obtained, manufactured, conveyed, and reprocessed using renewable energy.

4. Processed using sanitized manufacturing machines.

5. Produced from materials which are harmless and safe.

6. Physically designed to optimize material and energy.

7. Using biotic and industrial closed-loop sequences.

With the growing consciousness of customers with environmental issues, people favor the packaging that is manufactured from sustainable materials. Many countries are introducing biomaterials-based packaging into their markets. Competition among the manufacturers forces them to shift to totally sustainable packaging for safe survival in their competitive markets. The manufacturers are struggling to produce extraordinarily, high-quality materials that are sustainable and energy efficient. Leading retailers in the industries, for example, "Unilever" is committed to producing sustainable packaging products to reduce the problem of plastic waste in our daily life (Bavcon Kralj et al. 2020). A Swedish company, "Billerud", is manufacturing bio-based packaging material and aims to create sustainable development of the earth's resources by working with natural and renewable materials (Mülhaupt 2013).

\section{Principle of sustainable packaging}

Sustainable packaging is a composite theory which involves studies and certification to assess the packaging design, material assortment, treating, and life span (Yam and Lee 2012; Zhang et al. 1997). The purpose of sustainable packaging is to combine useful and advanced ingredients in packaging that stimulate cost-effective and ecological wellbeing. Packaging sustainability is frequently well-thought-out to be an advertising tool for supporting a distinctive new packing material. Packaging sustainability is crucial for organizations, such as the organization of sustainable packaging coalitions in the USA. The sustainable packaging alliance described sustainable packaging based on the literature review with four major criteria (Lewis et al. 2010). Firstly, the use of sustainable materials is crucial. The materials intended for the bundling must help reasonable advancement, while adequately ensuring the nature of the items. Normally, materials for sustainable packaging are manufactured to fulfill rules and regulation according to economics, material durability, and their demand in the market (Ljungberg 2007). The development of the material starts with a proper choice of the material, its performance, and the impact on the life cycle of the material. The second criterion of the sustainable packing is to meet the effective recovery challenge of material to reduce waste (Davis and Song 2006) along with the biological recovery, recycling, and energy recovery (Ljungberg 2007; Davis and Song 2006). The third norm of sustainable packaging includes the manufacturing of such sort of material, which is durable and has reusability for several times with minimum degradation in properties and the manufacturing of contaminant-free material. The last norm of sustainable packing is to use clean material that is safe and sound for human beings along with the safe removal of hazard materials associated with such a process (Zhang et al. 1997).

\section{Cellulosic fibers in the sustainable packaging}

Cellulose has excellent mechanical properties with low weight and biodegradable nature that possess high mechanical strength and stiffness, along with the best barrier resistant properties that can be used in the manufacturing of paper and biocomposites. Cellulose fiber-based packaging materials do not have any effect on medicines or pharmaceuticals that are packed in it. They are also harmless to a human's body. They are, in fact, green materials for food packaging and pharmaceutical industries. Properly manufactured composites, according to its end use, ensure a cleaner environment and harmless situations for human beings. 
Table 7 Available bio-nanocomposites and their properties in food packaging application

\begin{tabular}{|c|c|c|}
\hline Bio-nanocomposites & Properties & References \\
\hline Alginate/nanosilver & $\begin{array}{l}\text { Spoilage reduction } \\
\text { Lower water loss } \\
\text { Improvement of sensory attributes }\end{array}$ & Jiang et al. (2013) \\
\hline Corn starch/talc nanoparticles & $\begin{array}{l}\text { Increased Young modulus } \\
\text { Increased yield stress } \\
\text { Reduction in water permeability } \\
\text { Reduction in oxygen permeability }\end{array}$ & López et al. (2015) \\
\hline Calcium alginate/zinc oxide nanoparticles & Reduction in microbial load & Akbar and Anal (2014) \\
\hline Chitosan/polyvinyl alcohol/titanium nanoparticles & $\begin{array}{l}\text { Antibacterial activity against algae and bacteria } \\
\text { Extended shelf life }\end{array}$ & Youssef et al. (2015) \\
\hline Whey protein isolate/montmorillonite & $\begin{array}{l}\text { Increased tensile strength } \\
\text { Reduced swelling }\end{array}$ & Wakai and Almenar (2015) \\
\hline Polyvinylpyrrolidine/silver nanoparticles & $\begin{array}{l}\text { Edible coating } \\
\text { Greener colour } \\
\text { Lower water loss } \\
\text { Hindered growth of microorganisms }\end{array}$ & An et al. (2008) \\
\hline Soy protein isolate/montmorillonite & $\begin{array}{l}\text { Thermal stability } \\
\text { Improvement of mechanical properties } \\
\text { Water vapor permeability due to montmorillonite }\end{array}$ & Kumar et al. (2010) \\
\hline $\begin{array}{l}\text { Nanoemulsion coating of chitosan and mandarin essential } \\
\text { oil }\end{array}$ & Reduction of population of microorganisms & Donsì et al. (2015) \\
\hline Silver nanoparticles-based cellulose absorbent pads & $\begin{array}{l}\text { Reduction in population of aerobic bacteria } \\
\text { Reduction in microbial activity } \\
\text { Retarded the senescence of fruits } \\
\text { Gave juice appearance }\end{array}$ & Fernandez et al. (2010) \\
\hline Sodium alginate solution containing silver nanoparticles & $\begin{array}{l}\text { Antibacterial film } \\
\text { Reduced weight loss } \\
\text { Reduced soluble protein loss } \\
\text { Increased shelf life }\end{array}$ & Mohammed Fayaz et al. (2009) \\
\hline Nanozinc oxide neem-oil chitosan & Enhanced hindrance of bacterial activity & Sanuja et al. (2015) \\
\hline Starch/halloysite/nisin & $\begin{array}{l}\text { Improved mechanical properties } \\
\text { Improved antimicrobial activity }\end{array}$ & Meira et al. (2016) \\
\hline Wheat-gluten lignin nanoparticles & $\begin{array}{l}\text { Reduced water absorption ability } \\
\text { Improved thermal stability } \\
\text { Improved tensile strength }\end{array}$ & Yang et al. (2015) \\
\hline Pectin/nanohybrid layered double hydroxide salicylate & $\begin{array}{l}\text { Improved elongation at break } \\
\text { Improved water vapor barrier property } \\
\text { Extended shelf life }\end{array}$ & Gorrasi and Bugatti (2016) \\
\hline Tilapia skin gelatin hydrophilic-hydrophobic nanoclays & Improved mechanical/barrier properties & Nagarajan et al. (2014) \\
\hline
\end{tabular}

\section{Design process}

In the field of product manufacturing technology, good design is crucial. A product designed by expert professionals has different sets of quality and durability. To design a product of known quality, it may require various engineering principles and reproducibility. The end use of the product defines how much we should invest to claim its consistent quality. This is a very technical and sensitive step for any development in a product (Fricke 1996). The first step in designing a product of effective quality with biodegradable nature is the planning. Planning of design will decide the optimum cost, quality of the product, and its recyclability with minimum effect on the environment. Bieniawaski et al.
(1992) reported a series of steps with coherent classification to design a sustainable packaging product of defined quality. Figure 7 shows the design methodology adopted for sustainable packaging.

\section{Definition of the problem statement}

The definition of the problem statement is known to be the first step in any design process (Chikofsky and Cross 1990). Regarding the problems that could happen in the early design stages of the process for the cellulose nanofibers sustainable packaging products. The definition incorporates point-to-point data on cellulose nanofibers generation from natural fibers and its application towards sustainable 
packaging. In the later step, pertinent data on existing material other than cellulose nanofibers should be collected to create issue identification. The problem statement must address the difficulties and limitations associated with the packaging. Then finally, the criteria for success should be established. In this step, a conceivable solution should be considered for the problem statement.

Though the final design should be finalized based on some criteria to produce nanocellulosic material for biodegradable sustainable packaging, the following criteria should be followed: (1) the process should be economic and environmentally friendly, such as the process of cellulose nanofibers production for the bio-based sustainable packaging should be user-friendly and automatic with minimum interference by humans. (2) The produced cellulose nanofibers should have high mechanical and chemical properties, for this purpose, a process which can deliver high properties is hydrodynamic alignment by the flow-focusing concept. (3) The product (cellulose nanofibers) should be fully biodegradable and renewable. However, other parameters in terms of mechanical strength and good chemical stability are very necessary. From the above discussion, we conclude that there is still a lot of work that has to do for the production of biodegradable, environmentally friendly and high mechanical strength sustainable cellulose nanofibers.

\section{Collect specific information to eliminate the ambiguities}

The required information on physical, chemical, conductive strength, and stiffness of cellulose nanofibers should be collected. This should be performed based on the kind of material to be packed, either the food or non-food, temperature-sensitive medicine or consumer specified product. The nature of the product and its end-use application will define its required packaging specifications (Kalia et al. 2011). The engineering design of cellulose nanofibers is uncertain; the core reason for this uncertainty is that the design is performed without a holistic study survey. To develop biodegradable, eco-friendly cellulose nanofibers based on sustainable packaging products, we have to study the whole scenario starting from raw material till to the properties of the product to be packed. The proper knowledge about the properties of the raw material, source of the packing material, its contact time and temperature with the item to be packed, the shelf life of a product, and the chemical specifications of the product is a very time-consuming and challenging task. The complete study before designing the packaging product will ensure the delivery of the product to the customer without any compromise in the desired quality of the product (Gonzalez et al. 2011; Gon et al. 2012).

\section{The plainness of the design methodology adopted}

The objective of the plainness of the design procedure is to eliminate the ambiguities and mistakes during product design. It should be as straight forward because the holistic design factors are being considered while designing the packaging product (Benavides 2011). The design procedure will be adopted based on the likely output of the study design procedure. A recent study explains the freeze-drying procedure for the manufacturing of cellulose nanofibers used in the air filters (Nemoto et al. 2015). Nonetheless, there is a lot of work to do in the field of cellulose nanofibers-based sustainable packaging.

\section{Optimization of the design procedure}

This step involves adjusting the procedure according to the available sources to make it happen. Optimization minimizes and troubleshoots the risk involves in the design procedure. There are many methods of optimization available like linear optimization, linear least square optimization for different sorts of areas, including production, data plotting, and maintenance schedule. The holistic optimization of design procedure includes the economics of the process, limitation of resources; safety factors; renewability and recyclability; sustainability; and properties adjustment of the raw material. In the preparation of cellulose nanofibers, the optimization can be applied in the treatment process. The designer can perform statistics or mathematical optimization. Designers are using "Design of Experiments" to perform the optimization procedure while developing the product design process (Hossain et al. 2012).

\section{Execution of the design procedure}

Execution of the design procedure is the final and most sensitive stage. The process design should fulfill the criteria

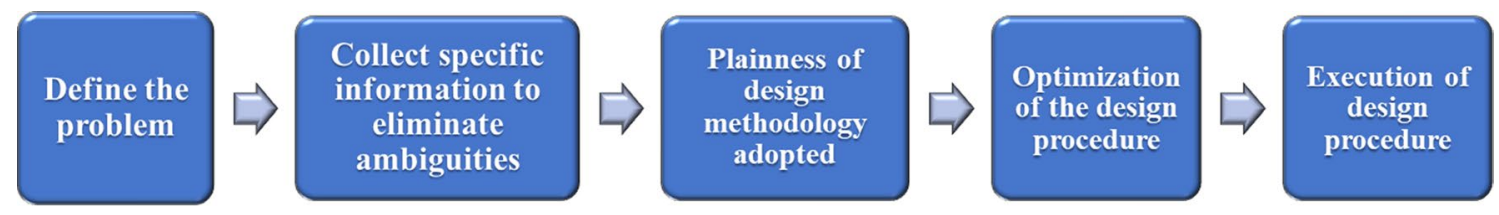

Fig. 7 Engineering design process of cellulose nanofibers for sustainable packaging applications via defining the problem, collecting specific information, the plainness of design methodology, optimization of the design procedures, and finally executing the design procedure 
for targeted success. The failure to do so will declare the design an invalid practice (Benavides 2011). So, it demands plenty of revision and attention to reprise the design procedure carefully. The approach that is adopted while designing the procedure should be robust and valid concerning practical manners. The design input information should be comprehensive for parameters and limitations defined while designing the procedure. The validity analysis of the procedure must be performed in order to avoid any failure while implementing a design procedure.

\section{Importance of designing the cellulose nanofibers}

Creative methodologies utilizing cellulose nanofibers can be helpful for the development of ecological and biodegradable sustainable packaging. The scientific design of cellulose nanofibers with measured qualitative and quantitative aspects can guarantee a sustainable packaging approach for the coming era (Mishra et al. 2012). The scientific design of cellulose nanofibers will ensure the efficient production process and its recycling. The most important and promising prose is that we can manage its waste by recycling (Davis and Song 2006; Marsh and Bugusu 2007). The specific engineering design of cellulose nanofibers according to target application will be helpful to apply known limitations concerning the environment, recycling, and sustainability of the packaging product. The well-known engineering design of the product can be helpful for the researcher to launch an efficient, sustainable packaging product with a targeted approach that can compete for the market.

\section{Engineering design challenges}

The basic approach of sustainable product manufacturing is the development of bio-based material with optimized cost and a high-quality product. This is a basic challenge on which the holistic product design process works. To meet this challenge, many factors must be considered while doing research and development on cellulose-based specific packaging products. Some of these factors are the durability of the product, technology adopted to manufacture the product, sustainability, and recyclability. The target market and enduse application limit these factors because the cost-efficient product can compete in the market. Engineering design is primarily important to manufacture the cost-efficient and sustainable cellulose-based product (Vargas et al. 2012).

The research and development on a pilot scale have been conducted to utilize cellulose-based material in sustainable packaging applications. The research on the laboratory-scale explores the current problems and their solutions before launching the idea on an industrial scale. Still, there is a need to perform research and planning on a small scale with the help of technical experts and material engineers to analyze the problems and for their appropriate solutions, so that cellulose can be utilized in different areas of biodegradable and sustainable packaging applications (Poole and Simon 1997). The pollution-free environment with minimum waste is the hot topic of the present age.

\section{Conclusion}

The utilization of nanocomposites comprised of abundant and biodegradable fillers and polymers has opened new avenues for both academia and industry to make sustainable products that could replace conventional synthetic materials causing environmental pollution. Nanocomposites, especially those which are reinforced with cellulose (which is known as the most abundant biopolymer on the sphere), have been highlighted as a significant replacement of synthetic products. Transformation of cellulose into nanoparticles through acidic, alkaline, 2,2,6,6-tetramethylpiperidine-1-oxyl and other methods (mechanical and biological) have enriched many properties, such as tensile strength, barrier, thermal and optical properties, and specific surface area; which increased from $51 \mathrm{~m}^{2} \mathrm{~g}^{-1}$ (cellulose nanofibers) to $533 \mathrm{~m}^{2} \mathrm{~g}^{-1}$ (cellulose nanocrystals). However, the energy-intensive and costly isolation process of cellulose nanoparticles caused hindrance in its utilization. Among various techniques used for the preparation of cellulosic nanocomposites, melt intercalation, solvent casting, in-situ polymerization, composite extrusion, and casting were found to be the most efficient and simple techniques that had a form of practicality to allow its adoption in an industry setting. Besides these processing techniques, the melt blending (through extruders) approach has been used quite a few times and proved a simple and efficient technique and has the potential to be used at the commercial level. Regardless of these, compatibility issues of hydrophilic filler (nanocellulose) with hydrophobic polymer and dispersion of filler in polymer matrices are some of the flaws of these materials. Practical and proper designing of the whole process was highlighted as potential future work and should be necessary for the identification of possibilities, suitability, and limitations of nanocomposites for food packaging applications. The utilization of cellulosic nanocomposites in food packaging applications has shown potential by improving barrier properties (189.61-0.1799 $\mathrm{cm}^{3} \mu \mathrm{m} / \mathrm{m}^{2} \mathrm{~d} \mathrm{kPa}$ ), water permeability $\left(6.3 \mathrm{~g} \mathrm{~mm} \mathrm{~m}^{-2}\right.$ day $\mathrm{kPa}$ (without cellulose nanocrystals) to $4.7 \mathrm{~g} \mathrm{~mm} \mathrm{~m}^{-2}$ day $\mathrm{kPa}$ (with $1 \mathrm{wt} \%$ cellulose nanocrystals), and mechanical properties (Young modulus of cellulose nanocomposite increased $87 \%$ as compared to pure polypropylene), which suggests that in near future nanocomposites made from biopolymers will start to replace conventional synthetic plastic products only if: 
- The isolation process of cellulose and its conversion into nanoparticles would be cost and energy effective.

- The variability of extracted fibers properties should be reduced.

- The compatibility issues between hydrophobic natural fibers (cellulose) and hydrophilic polymers would be solved.

- Adhesive agents, coupling agents or compatibilizers should be developed from renewable sources.

- Life cycle assessment and biodegradability of nanocomposites should be properly researched.

- New processing technologies should be developed.

Acknowledgements DR and AO would like to acknowledge the support given by the EPSRC Project "Advancing Creative Circular Economies for Plastics via Technological-Social Transitions" (ACCEPT Transitions, EP/S025545/1). AO wishes to acknowledge the support of The Bryden Centre Project (Project ID VA5048), which was awarded by The European Union's INTERREG VA Programme.

\section{Funding}

\section{Compliance with ethical standards}

Conflict of interest The author declares no competing financial interests.

Open Access This article is licensed under a Creative Commons Attribution 4.0 International License, which permits use, sharing, adaptation, distribution and reproduction in any medium or format, as long as you give appropriate credit to the original author(s) and the source, provide a link to the Creative Commons licence, and indicate if changes were made. The images or other third party material in this article are included in the article's Creative Commons licence, unless indicated otherwise in a credit line to the material. If material is not included in the article's Creative Commons licence and your intended use is not permitted by statutory regulation or exceeds the permitted use, you will need to obtain permission directly from the copyright holder. To view a copy of this licence, visit http://creativecommons.org/licenses/by/4.0/.

\section{References}

Abdollahi M et al (2013) Reducing water sensitivity of alginate bio-nanocomposite film using cellulose nanoparticles. Int J Biol Macromol 54:166-173. https://doi.org/10.1016/j.ijbio mac.2012.12.016

Abraham E et al (2012) X-ray diffraction and biodegradation analysis of green composites of natural rubber/nanocellulose. Polym Degrad Stab 97:2378-2387. https://doi.org/10.1016/j.polym degradstab.2012.07.028

Akbar A, Anal AK (2014) Zinc oxide nanoparticles loaded active packaging, a challenge study against Salmonella typhimurium and Staphylococcus aureus in ready-to-eat poultry meat. Food Control 38:88-95. https://doi.org/10.1016/j.foodcont.2013.09.065

Alemdar A, Sain M (2008) Isolation and characterization of nanofibers from agricultural residues-Wheat straw and soy hulls.
Biores Technol 99:1664-1671. https://doi.org/10.1016/j.biort ech.2007.04.029

Al-Tayyar NA et al (2020a) Antimicrobial packaging efficiency of $\mathrm{ZnO}-\mathrm{SiO} 2$ nanocomposites infused into PVA/CS film for enhancing the shelf life of food products. Food Packaging and Shelf Life. 25:100523. https://doi.org/10.1016/j.fpsl.2020.100523

Al-Tayyar NA et al (2020b) Antimicrobial food packaging based on sustainable Bio-based materials for reducing foodborne Pathogens: a review. Food Chem 310:125915. https://doi. org/10.1016/j.foodchem.2019.125915

An J et al (2008) Physical, chemical and microbiological changes in stored green asparagus spears as affected by coating of silver nanoparticles-PVP. LWT-Food Science and Technology. 41:1100-1107. https://doi.org/10.1016/j.1wt.2007.06.019

Andresen $\mathrm{M}$ et al (2007) Nonleaching antimicrobial films prepared from surface-modified microfibrillated cellulose. Biomacromolecules 8:2149-55. https://doi.org/10.1021/bm070304e

Araki J et al (1999) Influence of surface charge on viscosity behavior of cellulose microcrystal suspension. Journal of wood science. 45:258-261. https://doi.org/10.1007/BF01177736

Aulin C et al (2010) Oxygen and oil barrier properties of microfibrillated cellulose films and coatings. Cellulose 17:559-574. https ://www.ncbi.nlm.nih.gov/pubmed/22976562

Aulin C et al (2012) High strength, flexible and transparent nanofibrillated cellulose-nanoclay biohybrid films with tunable oxygen and water vapor permeability. Nanoscale. 4:6622-6628. https://doi. org/10.1039/c2nr31726e

Azeredo HM et al (2010) Nanocellulose reinforced chitosan composite films as affected by nanofiller loading and plasticizer content. J Food Sci 75:N1-N7. https://doi.org/10.111 1/j.1750-3841.2009.01386.x

Azizi Samir MAS et al (2004) Preparation of cellulose whiskers reinforced nanocomposites from an organic medium suspension. Macromolecules 37:1386-1393. https://doi.org/10.1021/ma030 $532 \mathrm{a}$

Bandyopadhyay-Ghosh S et al (2015) The use of biobased nanofibres in composites. Biofiber Reinforcements in Composite Materials. Elsevier, 2015, pp 571-647. https://doi.org/10.1533/97817 82421276.5.571

Bavcon Kralj M et al (2020) Dish handwashing: an overlooked source of contamination. Environ Chem Lett 18:181-185. https://doi. org/10.1007/s10311-019-00918-5

Benavides EM (2011) Advanced engineering design: an integrated approach. Elsevier. https://doi.org/10.5923/j.textile.20120106.05

Bharimalla A et al (2017) Nanocellulose-polymer composites for applications in food packaging: Current status, future prospects and challenges. Polym Plast Technol Eng 56:805-823. https://doi. org/10.1080/03602559.2016.1233281

Bharimalla A et al (2019) Nanocellulose-polymer composites: novel materials for food packaging applications. Polymers for agrifood applications. Springer, Berlin, pp. 553-599. https://doi. org/10.1007/978-3-030-19416-1_27

Bideau B et al (2016) Mechanical and antibacterial properties of a nanocellulose-polypyrrole multilayer composite. Mater Sci Eng, C 69:977-984. https://doi.org/10.1016/j.msec.2016.08.005

Bieniawski Z (1992) Principles of engineering design for rock mechanics. In: The 33th US Symposium on Rock Mechanics (USRMS). American Rock Mechanics Association, 1992. https://doi. org/10.1007/s00603-019-01962-x

Bilbao-Sainz C et al (2011) HPMC reinforced with different cellulose nano-particles. Carbohyd Polym 86:1549-1557. https://doi. org/10.1016/j.carbpol.2011.06.060

Boumail A et al (2013) Characterization of trilayer antimicrobial diffusion films (ADFs) based on methylcellulose-polycaprolactone composites. J Agric Food Chem 61:811-21. https://doi. org/10.1021/jf304439s 
Bras J et al (2010) Mechanical, barrier, and biodegradability properties of bagasse cellulose whiskers reinforced natural rubber nanocomposites. Ind Crops Prod 32:627-633. https://doi. org/10.1016/j.indcrop.2010.07.018

Bras J et al (2011) Correlation between stiffness of sheets prepared from cellulose whiskers and nanoparticles dimensions. Carbohyd Polym 84:211-215. https://doi.org/10.1016/j.carbp ol.2010.11.022

Campos CA et al (2011) Development of edible films and coatings with antimicrobial activity. Food Bioprocess Technol 4:849-875. https ://doi.org/10.1007/s11947-010-0434-1

Cao X et al (2007) New nanocomposite materials reinforced with flax cellulose nanocrystals in waterborne polyurethane. Biomacromol 8:899-904. https://doi.org/10.1021/bm0610368

Cao X et al (2008) Starch-based nanocomposites reinforced with flax cellulose nanocrystals. Express polymer letters. 2:502-510. https ://doi.org/10.3144/expresspolymlett.2008.60

Cazon P, Vazquez M (2020) Mechanical and barrier properties of chitosan combined with other components as food packaging film. Environ Chem Lett 18:257-267. https://doi.org/10.1007/ s10311-019-00936-3

Cerqueira $\mathrm{M}$ et al (2011) Galactomannans use in the development of edible films/coatings for food applications. Trends Food Sci Technol 22:662-671. https://doi.org/10.1016/j.tifs.2011.07.002

Chen $\mathrm{H}$ et al (2019) Upcycling food waste digestate for energy and heavy metal remediation applications. Resour Conserv Recycl X. 3:100015. https://doi.org/10.1016/j.rcrx.2019.100015

Chen Y et al (2009) Bionanocomposites based on pea starch and cellulose nanowhiskers hydrolyzed from pea hull fibre: effect of hydrolysis time. Carbohyd Polym 76:607-615. https://doi. org/10.1016/j.carbpol.2008.11.030

Chen W et al (2011a) Isolation and characterization of cellulose nanofibers from four plant cellulose fibers using a chemical-ultrasonic process. Cellulose 18:433-442. https://doi. org/10.1007/s10570-011-9497-z

Chen W et al (2011b) Individualization of cellulose nanofibers from wood using high-intensity ultrasonication combined with chemical pretreatments. Carbohyd Polym 83:1804-1811. https ://doi.org/10.1016/j.carbpol.2010.10.040

Cheng KC et al (2011) Pullulan: biosynthesis, production, and applications. Appl Microbiol Biotechnol 92:29-44. https:// doi.org/10.1007/s00253-011-3477-y

Chikofsky EJ, Cross JH (1990) Reverse engineering and design recovery: a taxonomy. IEEE Softw 7:13-17. https://doi. org/10.1109/52.43044

Ching YC et al (2015) Preparation and characterization of polyvinyl alcohol-based composite reinforced with nanocellulose and nanosilica. BioResources 10:3364-3377. https://doi. org/10.15376/biores.10.2.3364-3377

Costa L et al (2015) Extraction and characterization of cellulose nanocrystals from corn stover. Cell Chem Technol 49:127-133. https://cellulosechemtechnol.ro/pdf/CCT2(2015)/p.127-133. pdf

Cruz-Romero M, Kerry J (2008) Crop-based biodegradable packaging and its environmental implications. CAB Rev. Perspect. Agric. Vet. Sci. Nutr. Nat. Resour. 3:1-25. https://doi. org/10.1079/PAVSNNR20083074

Cudjoe E et al (2017) Miscanthus Giganteus: a commercially viable sustainable source of cellulose nanocrystals. Carbohyd Polym 155:230-241. https://doi.org/10.1016/j.carbpol.2016.08.049

da Silva JB et al (2012) Cassava starch-based films plasticized with sucrose and inverted sugar and reinforced with cellulose nanocrystals. J Food Sci 77:N14-N19. https://doi.org/10.111 1/j.1750-3841.2012.02710.x
Darmadji P, Izumimoto M (1996) Effect of chitosan on meat preservation. Indonesian food and nutrition progress. 3:51-56. https ://doi.org/10.1016/0309-1740(94)90114-7

Davis G, Song J (2006) Biodegradable packaging based on raw materials from crops and their impact on waste management. Ind Crops Prod 23:147-161. https://doi.org/10.1016/j.inder op.2005.05.004

de Amorim JDP et al (2020) Plant and bacterial nanocellulose: production, properties and applications in medicine, food, cosmetics, electronics and engineering. A review. Environ Chem Lett 18:851-869. https://doi.org/10.1007/s10311-020-00989-9

De Menezes AJ et al (2009) Extrusion and characterization of functionalized cellulose whiskers reinforced polyethylene nanocomposites. Polymer 50:4552-4563. https://doi.org/10.1016/j.polym er.2009.07.038

de Mesquita JP et al (2012) Bio-based nanocomposites obtained through covalent linkage between chitosan and cellulose nanocrystals. Carbohyd Polym 90:210-217. https://doi. org/10.1016/j.carbpol.2012.05.025

Dehnad D et al (2014) Optimization of physical and mechanical properties for chitosan-nanocellulose biocomposites. Carbohydr Polym 105:222-8. https://doi.org/10.1016/j.carbpol.2014.01.094

Dhar P et al (2015) Poly (3-hydroxybutyrate)/cellulose nanocrystal films for food packaging applications: barrier and migration studies. Polym Eng Sci 55:2388-2395. https://doi.org/10.1002/ pen. 24127

Donsì $\mathrm{F}$ et al (2015) Green beans preservation by combination of a modified chitosan based-coating containing nanoemulsion of mandarin essential oil with high pressure or pulsed light processing. Postharvest Biol Technol 106:21-32. https://doi. org/10.1016/j.postharvbio.2015.02.006

Duarte EB et al (2015) Production of hydroxyapatite-bacterial cellulose nanocomposites from agroindustrial wastes. Cellulose 22:3177-3187. https://doi.org/10.1007/s10570-015-0734-8

Dufresne A (2013) Nanocellulose: a new ageless bionanomaterial. Mater Today 16:220-227. https://doi.org/10.1016/j.matto d.2013.06.004

Dufresne A (2017a) Nanocellulose: from nature to high performance tailored materials. Walter de Gruyter GmbH \& Co KG. https:// doi.org/10.1515/9783110254600

Dufresne A (2017b) Cellulose nanomaterial reinforced polymer nanocomposites. Curr Opin Colloid Interf Sci 29:1-8. https://doi. org/10.1016/j.cocis.2017.01.004

Dufresne A (2019) Nanocellulose processing properties and potential applications. Curr For Rep 5:76-89. https://doi.org/10.1007/ s40725-019-00088-1

Dufresne A et al (2000) Cellulose microfibrils from potato tuber cells: processing and characterization of starch-cellulose microfibril composites. J Appl Polym Sci 76:2080-2092. https://doi. org/10.1002/(SICI)1097-4628(20000628)76:14\%3C2080:AIDAPP12\%3E3.0.CO;2-U

El Achaby $M$ et al (2017) Processing and properties of eco-friendly bio-nanocomposite films filled with cellulose nanocrystals from sugarcane bagasse. Int J Biol Macromol 96:340-352. https://doi. org/10.1016/j.ijbiomac.2016.12.040

El Achaby M et al (2018) Reuse of red algae waste for the production of cellulose nanocrystals and its application in polymer nanocomposites. Int J Biol Macromol 106:681-691. https://doi. org/10.1016/j.ijbiomac.2017.08.067

El Miri N et al (2015) Bio-nanocomposite films reinforced with cellulose nanocrystals: rheology of film-forming solutions, transparency, water vapor barrier and tensile properties of films. Carbohydr Polym 129:156-67. https://doi.org/10.1016/j.carbp ol.2015.04.051

El-Sayed SM et al (2020) Rational design of chitosan/guar gum/zinc oxide bionanocomposites based on Roselle calyx extract for Ras 
cheese coating. Carbohyd Polym. https://doi.org/10.1016/j.carbp ol.2020.116234

Fatah I et al (2014) Exploration of a chemo-mechanical technique for the isolation of nanofibrillated cellulosic fiber from oil palm empty fruit bunch as a reinforcing agent in composites materials. Polymers 6:2611-2624. https://doi.org/10.3390/polym6102611

Fernandez A et al (2010) Cellulose-silver nanoparticle hybrid materials to control spoilage-related microflora in absorbent pads located in trays of fresh-cut melon. Int J Food Microbiol 142:222-228. https://doi.org/10.1016/j.ijfoodmicro.2010.07.001

Ferreira AR et al (2014) Biodegradable films produced from the bacterial polysaccharide FucoPol. Int J Biol Macromol. 71:111-6. https://doi.org/10.1016/j.ijbiomac.2014.04.022

Ferreira ARV et al (2016) Development and characterization of bilayer films of FucoPol and chitosan. Carbohydr Polym 147:8-15. https ://doi.org/10.1016/j.carbpol.2016.03.089

Ferrer A et al (2017) Nanocellulose in packaging: advances in barrier layer technologies. Ind Crops Prod 95:574-582. https://doi. org/10.1016/j.indcrop.2016.11.012

Follain N et al (2013) Water transport properties of bio-nanocomposites reinforced by Luffa cylindrica cellulose nanocrystals. J Membr Sci 427:218-229. https://doi.org/10.1016/j.memsc i.2012.09.048

Fortunati E et al (2012) Effects of modified cellulose nanocrystals on the barrier and migration properties of PLA nano-biocomposites. Carbohydr Polym 90:948-56. https://doi.org/10.1016/j.carbp ol.2012.06.025

Fortunati E et al (2013) Combined effects of cellulose nanocrystals and silver nanoparticles on the barrier and migration properties of PLA nano-biocomposites. J Food Eng 118:117-124. https:// doi.org/10.1016/j.jfoodeng.2013.03.025

Fortunati E et al (2014a) Nano-biocomposite films with modified cellulose nanocrystals and synthesized silver nanoparticles. Carbohydr Polym 101:1122-33. https://doi.org/10.1016/j.carbp ol.2013.10.055

Fortunati E et al (2014b) Investigation of thermo-mechanical, chemical and degradative properties of PLA-limonene films reinforced with cellulose nanocrystals extracted from Phormium tenax leaves. Eur Polym J 56:77-91. https://doi.org/10.1016/j.eurpo lymj.2014.03.030

Fortunati E et al (2016) Revalorization of barley straw and husk as precursors for cellulose nanocrystals extraction and their effect on PVA_CH nanocomposites. Ind Crops Prod 92:201-217. https ://doi.org/10.1016/j.indcrop.2016.07.047

Fricke G (1996) Successful individual approaches in engineering design. Res Eng Design 8:151-165. https://doi.org/10.1007/ BF01608350

Frone AN et al (2019) Thermal and mechanical behavior of biodegradable polyester films containing cellulose nanofibers. J Therm Anal Calorim 138:2387-2398. https://doi.org/10.1007/s 1097 3-019-08218-4

Fujisawa $S$ et al (2012) Superior reinforcement effect of TEMPO-oxidized cellulose nanofibrils in polystyrene matrix: optical, thermal, and mechanical studies. Biomacromolecules 13:2188-2194. https://doi.org/10.1021/bm300609c

Gaikwad KK et al (2018) Oxygen scavenging films in food packaging. Environ Chem Lett 16:523-538. https://doi.org/10.1007/s1031 $1-018-0705-\mathrm{z}$

Gaikwad KK et al (2019) Moisture absorbers for food packaging applications. Environ Chem Lett 17:609-628. https://doi.org/10.1007/ s10311-018-0810-z

Gaikwad KK et al (2020) Ethylene scavengers for active packaging of fresh food produce. Environ Chem Lett 18:269-284. https://doi. org/10.1007/s10311-019-00938-1
Galgano F (2015) Biodegradable packaging and edible coating for fresh-cut fruits and vegetables. Ital J Food Sci 27:1-20. https:// www.itjfs.com/index.php/ijfs

George J (2012) High performance edible nanocomposite films containing bacterial cellulose nanocrystals. Carbohyd Polym 87:2031-2037. https://doi.org/10.1016/j.carbpol.2011.10.019

George $\mathrm{J}$ et al (2014) Hybrid HPMC nanocomposites containing bacterial cellulose nanocrystals and silver nanoparticles. Carbohydr Polym. 105:285-92. https://doi.org/10.1016/j.carbp ol.2014.01.057

George J et al (2011) Bacterial cellulose nanocrystals exhibiting high thermal stability and their polymer nanocomposites. Int J Biol Macromol 48:50-57. https://doi.org/10.1016/j.ijbio mac.2010.09.013

Ghaderi M et al (2014) All-cellulose nanocomposite film made from bagasse cellulose nanofibers for food packaging application. Carbohydr Polym 104:59-65. https://doi.org/10.1016/j.carbp ol.2014.01.013

Gois GdS et al (2017) Soil Biodegradation of PLA/CNW Nanocomposites modified with ethylene oxide derivatives. Mater Res 20:899-904. http://dx.doi.org/10.1590/1980-5373-mr-2016-0960

Gon D et al (2012) Jute composites as wood substitute. International Journal of Textile Science. 1:84-93. https://doi.org/10.5923/j. textile.20120106.05

Gonzalez RW et al (2011) Thermo-mechanical pulping as a pretreatment for agricultural biomass for biochemical conversion. BioResour 6:1599-1614. http://ncsu.edu/bioresources

Gorrasi G, Bugatti V (2016) Edible bio-nano-hybrid coatings for food protection based on pectins and LDH-salicylate: preparation and analysis of physical properties. LWT Food Sci Technol 69:139145. https://doi.org/10.1016/j.lwt.2016.01.038

Gray N et al (2018) Influence of cellulose nanocrystal on strength and properties of low density polyethylene and thermoplastic starch composites. Ind Crops Prod 115:298-305. https://doi. org/10.1016/j.indcrop.2018.02.017

Gutiérrez TJ, Alvarez VA (2017a) Properties of native and oxidized corn starch/polystyrene blends under conditions of reactive extrusion using zinc octanoate as a catalyst. React Funct Polym 112:33-44. https://doi.org/10.1016/j.reactfunctpolym .2017.01.002

Gutiérrez TJ, Alvarez VA (2017b) Eco-friendly films prepared from plantain flour/PCL blends under reactive extrusion conditions using zirconium octanoate as a catalyst. Carbohydr Polym 178:260-269. https://doi.org/10.1016/j.carbpol.2017.09.026

Habibi Y, Vignon MR (2008) Optimization of cellouronic acid synthesis by TEMPO-mediated oxidation of cellulose III from sugar beet pulp. Cellulose 15:177-185. https://doi.org/10.1007/s1057 $0-007-9179-\mathrm{z}$

Hassan $\mathrm{N}$ et al (2020) Biofuels and renewable chemicals production by catalytic pyrolysis of cellulose: a review. Environ Chem Lett. https://doi.org/10.1007/s10311-020-01040-7

Helbert W et al (1996) Thermoplastic nanocomposites filled with wheat straw cellulose whiskers. Part I: processing and mechanical behavior. Polym Compos 17:604-611. https://doi.org/10.1002/ pc. 10650

Henriksson $M$ et al (2007) An environmentally friendly method for enzyme-assisted preparation of microfibrillated cellulose (MFC) nanofibers. Eur Polymer J 43:3434-3441. https://doi. org/10.1016/j.eurpolymj.2007.05.038

Hiasa $S$ et al (2014) Isolation of cellulose nanofibrils from mandarin (Citrus unshiu) peel waste. Ind Crops Prod 62:280-285. https:// doi.org/10.1016/j.indcrop.2014.08.007

Hossain MS et al (2012) Selective extraction, separation and recovery of $\mathrm{Cu}$ (II) in presence of $\mathrm{Zn}$ (II) and Ni (II) from leach liquor of waste printed circuit board using microcapsules coated 
with Cyanex 272. Korean J Chem Eng 29:668-675. https://doi. org/10.1007/s11814-011-0220-4

Hult E-L et al (2010) Efficient approach to high barrier packaging using microfibrillar cellulose and shellac. Cellulose 17:575-586. https ://doi.org/10.1007/s10570-010-9408-8

Huq $\mathrm{T}$ et al (2012) Nanocrystalline cellulose (NCC) reinforced alginate based biodegradable nanocomposite film. Carbohydr Polym 90:1757-63. https://doi.org/10.1016/j.carbpol.2012.07.065

Isogai A et al (2011) TEMPO-oxidized cellulose nanofibers. Nanoscale 3:71-85. https://doi.org/10.1039/c0nr00583e

Iwamoto $S$ et al (2011) Structure and mechanical properties of wetspun fibers made from natural cellulose nanofibers. Biomacromol 12:831-836. https://doi.org/10.1021/bm101510r

Iwatake A et al (2008) Cellulose nanofiber-reinforced polylactic acid. Composites Science and Technology. 68:2103-2106

Jiang $\mathrm{T}$ et al (2013) Effect of alginate/nano-Ag coating on microbial and physicochemical characteristics of shiitake mushroom (Lentinus edodes) during cold storage. Food Chem 141:954-60. https ://doi.org/10.1016/j.foodchem.2013.03.093

Jo C et al (2001) Quality properties of pork sausage prepared with water-soluble chitosan oligomer. Meat Sci 59:369-375. https:// doi.org/10.1016/s0309-1740(01)00089-4

Jonoobi M et al (2010) Mechanical properties of cellulose nanofiber (CNF) reinforced polylactic acid (PLA) prepared by twin screw extrusion. Composites Science and Technology. 70:1742-1747. https://doi.org/10.1016/j.compscitech.2010.07.005

Kalia $S$ et al (2011) Cellulose-based bio-and nanocomposites: a review. International Journal of Polymer Science. https://doi. org/10.1155/2011/837875

Kanoth BP et al (2015) A cost-effective method to prepare cellulose nanofiber from coir. Adv Sci Eng Med 7:492-497. https://doi. org/10.1166/asem.2015.1721

Kargarzadeh $\mathrm{H}$ et al (2017) Handbook of nanocellulose and cellulose nanocomposites. Wiley Online Library. https://doi. org/10.1002/9783527689972

Khalil HA et al (2016) A review on nanocellulosic fibres as new material for sustainable packaging: process and applications. Renew Sustain Energy Rev 64:823-836. https://doi. org/10.1016/j.rser.2016.06.072

Khalil HA et al (2017) Seaweed based sustainable films and composites for food and pharmaceutical applications: a review. Renew Sustain Energy Rev 77:353-362. https://doi.org/10.1016/j. rser.2017.04.025

Khan RA et al (2010) Production and properties of nanocellulosereinforced methylcellulose-based biodegradable films. J Agric Food Chem 58:7878-85. https://doi.org/10.1021/jf1006853

Khan A et al (2012) Mechanical and barrier properties of nanocrystalline cellulose reinforced chitosan based nanocomposite films. Carbohydr Polym 90:1601-8. https://doi.org/10.1016/j. carbpol.2012.07.037

Khan RA et al (2013) Mechanical and barrier properties of nanocrystalline cellulose reinforced poly (caprolactone) composites: effect of gamma radiation. J Appl Polym Sci 129:3038-3046. https://doi.org/10.1002/app.38896

Khan A et al (2014) Genipin cross-linked nanocomposite films for the immobilization of antimicrobial agent. ACS Appl Mater Interfaces 6:15232-42. https://doi.org/10.1021/am503564m

Khoshkava V, Polypropylene (PP) nanocomposites incorporating nanocrystalline cellulose (NCC).McGill University Libraries, 2014. https://www.semanticscholar.org/paper/Polypropylene(PP)-nanocomposites-incorporating-Khoshkava/757406c3db bc6c8a324be9a4144d073e87c04486

Kumar P et al (2010) Preparation and characterization of bio-nanocomposite films based on soy protein isolate and montmorillonite using melt extrusion. J Food Eng 100:480-489. https:// doi.org/10.1016/j.jfoodeng.2010.04.035
Kumar R et al (2019) Effect of nano-cellulosic fiber on mechanical and barrier properties of polylactic acid (PLA) green nanocomposite film. Materials Research Express. 6:125108. https://doi. org/10.1088/2053-1591/ab5755

Kuswandi B (2017) Environmental friendly food nano-packaging. Environ Chem Lett 15:205-221. https://doi.org/10.1007/s1031 1-017-0613-7

Lasseuguette E et al (2008) Rheological properties of microfibrillar suspension of TEMPO-oxidized pulp. Cellulose 15:425-433. https://doi.org/10.1007/s10570-007-9184-2

Lavoine $\mathrm{N}$ et al (2014) Microfibrillated cellulose coatings as new release systems for active packaging. Carbohydr Polym 103:528-537. https://doi.org/10.1016/j.carbpol.2013.12.035

Lavoine $\mathrm{N}$ et al (2012) Microfibrillated cellulose-Its barrier properties and applications in cellulosic materials: a review. Carbohyd Polym 90:735-764. https://doi.org/10.1016/j.carbp ol.2012.05.026

Lewis $\mathrm{H}$ et al (2010) Evaluating the sustainability impacts of packaging: the plastic carry bag dilemma. Packaging Technology and Science: An International Journal. 23:145-160. https:// doi.org/10.1002/pts.886

$\mathrm{Li} F$ et al (2015) The potential of nanocellulose in the packaging field: a review. Packag Technol Sci 28:475-508. https://doi.org/10.1002/ pts. 2121

Li C et al (2019) Diffusive gradients in thin films: devices, materials and applications. Environ Chem Lett 17:801-831. https://doi. org/10.1007/s10311-018-00839-9

Li Q et al (2009) Structure and properties of the nanocomposite films of chitosan reinforced with cellulose whiskers. J Polym Sci, Part B: Polym Phys 47:1069-1077. https://doi.org/10.1002/polb.21711

$\mathrm{Li} \mathrm{F}$ et al (2013) Multi-functional coating of cellulose nanocrystals for flexible packaging applications. Cellulose 20:2491-2504. https ://doi.org/10.1007/s 10570-013-0015-3

Li M-C et al (2015) Cellulose nanoparticles: structure-morphologyrheology relationships. ACS Sustainable Chemistry \& Engineering. 3:821-832. https://doi.org/10.1021/acssuschemeng.5b00144

Lin N, Dufresne A (2014) Surface chemistry, morphological analysis and properties of cellulose nanocrystals with gradiented sulfation degrees. Nanoscale 6:5384-93. https://doi.org/10.1039/ c3nr06761k

Ljungberg LY (2007) Materials selection and design for development of sustainable products. Mater Des 28:466-479. https://doi. org/10.1016/j.matdes.2005.09.006

López OV et al (2015) Food packaging bags based on thermoplastic corn starch reinforced with talc nanoparticles. Food Hydrocoll 43:18-24. https://doi.org/10.1016/j.foodhyd.2014.04.021

Lu P, Hsieh Y-L (2012) Cellulose isolation and core-shell nanostructures of cellulose nanocrystals from chardonnay grape skins. Carbohyd Polym 87:2546-2553. https://doi.org/10.1016/j.carbp ol.2011.11.023

$\mathrm{Lu}$ Y et al (2006) Morphological, thermal and mechanical properties of ramie crystallites-reinforced plasticized starch biocomposites. Carbohyd Polym 63:198-204. https://doi.org/10.1016/j.carbp ol.2005.08.027

Luzi F et al (2017) Cellulose nanocrystals from Actinidia deliciosa pruning residues combined with carvacrol in PVA_CH films with antioxidant/antimicrobial properties for packaging applications. Int J Biol Macromol 104:43-55. https://doi.org/10.1016/j.ijbio mac.2017.05.176

Marimuthu TS, Atmakuru R (2015) Isolation and characterization of cellulose nanofibers from the aquatic weed Water Hyacinth: Eichhornia crassipes. In: Handbook of polymer nanocomposites. Processing, performance and application. Springer, Berlin, 2015, pp 37-46. https://doi.org/10.1007/978-3-642-45232-1_55 
Marsh K, Bugusu B (2007) Food packaging—roles, materials, and environmental issues. J Food Sci 72:R39-R55. https://doi.org/1 0.1111/j.1750-3841.2007.00301.x

Matos Ruiz M et al (2000) Processing and characterization of new thermoset nanocomposites based on cellulose whiskers. Compos Interfaces 7:117-131. https://doi.org/10.1163/1568554003 00184271

Meira SMM et al (2016) Starch-halloysite nanocomposites containing nisin: characterization and inhibition of Listeria monocytogenes in soft cheese. LWT-Food Sci Technol 68:226-234. https://doi. org/10.1016/j.lwt.2015.12.006

Merino D et al (2019a) Potential agricultural mulch films based on native and phosphorylated corn starch with and without surface functionalization with chitosan. J Polym Environ 27:97-105. https://doi.org/10.1007/s10924-018-1325-1

Merino D et al (2019) Structural and thermal properties of agricultural mulch films based on native and oxidized corn starch nanocomposites. Starch-Stärke. https://doi.org/10.1002/star.201800341

Mishra SP et al (2012) Production of nanocellulose from native cellulose-various options utilizing ultrasound. BioResour 7:04220436. http://ncsu.edu/bioresources

Mohamed M et al (2015) Physicochemical properties of "green" nanocrystalline cellulose isolated from recycled newspaper. Rsc Advances. 5:29842-29849. https://doi.org/10.1039/ C4RA17020B

Mohammed Fayaz A et al (2009) Mycobased synthesis of silver nanoparticles and their incorporation into sodium alginate films for vegetable and fruit preservation. J Agric Food Chem 57:6246-6252. https://doi.org/10.1021/jf900337h

Morin-Crini N et al (2019) Applications of chitosan in food, pharmaceuticals, medicine, cosmetics, agriculture, textiles, pulp and paper, biotechnology, and environmental chemistry. Environ Chem Lett. https://doi.org/10.1007/s10311-019-00904-x

Mülhaupt R (2013) Green polymer chemistry and bio-based plastics: dreams and reality. Macromol Chem Phys 214:159-174. https ://doi.org/10.1002/macp.201200439

Nagarajan M et al (2014) Characteristics of bio-nanocomposite films from tilapia skin gelatin incorporated with hydrophilic and hydrophobic nanoclays. J Food Eng 143:195-204. https://doi. org/10.1016/j.jfoodeng.2014.06.038

Nakagaito AN, Yano H (2008) The effect of fiber content on the mechanical and thermal expansion properties of biocomposites based on microfibrillated cellulose. Cellulose 15:555-559. https://doi.org/10.1007/s10570-008-9212-x

Nemoto J et al (2015) Simple freeze-drying procedure for producing nanocellulose aerogel-containing, high-performance air filters. ACS Appl Mater Interfaces 7:19809-19815. https:// doi.org/10.1021/acsami.5b05841

Neto WPF et al (2013) Extraction and characterization of cellulose nanocrystals from agro-industrial residue-Soy hulls. Ind Crops Prod 42:480-488. https://doi.org/10.1016/j.inder op.2012.06.041

Oksman K et al (2006) Manufacturing process of cellulose whiskers/ polylactic acid nanocomposites. Composites science and technology. 66:2776-2784. https://doi.org/10.1016/j.compscitec h.2006.03.002

Osman AI et al (2019) Reusing, recycling and up-cycling of biomass: a review of practical and kinetic modelling approaches. Fuel Process Technol 192:179-202. https://doi.org/10.1016/j.fupro c. 2019.04 .026

Osman AI et al (2020) Upcycling brewer's spent grain waste into activated carbon and carbon nanotubes for energy and other applications via two-stage activation. J Chem Technol Biotechnol 95:183-195. https://doi.org/10.1002/jctb.6220

Pääkkö M et al (2007) Enzymatic hydrolysis combined with mechanical shearing and high-pressure homogenization for nanoscale cellulose fibrils and strong gels. Biomacromol 8:1934-1941. https://doi.org/10.1021/bm061215p

Padervand $\mathrm{M}$ et al (2020) Removal of microplastics from the environment. A review. Environ Chem Lett 18:807-828. https://doi. org/10.1007/s10311-020-00983-1

Paralikar SA et al (2008) Poly (vinyl alcohol)/cellulose nanocrystal barrier membranes. J Membr Sci 320:248-258. https://doi. org/10.1016/j.memsci.2008.04.009

Patiño-Masó J et al (2019) TEMPO-oxidized cellulose nanofibers: a potential bio-based superabsorbent for diaper production. Nanomaterials. 9:1271. https://doi.org/10.3390/nano9091271

Pecoraro É et al (2007) Bacterial cellulose from Glucanacetobacter xylinus: preparation, properties and applications. Monomers, polymers and composites from renewable resources. Elsevier, 2007, pp 369-383. https://doi.org/10.1016/B978-0-08-04531 6-3.00017-X

Pereda M et al (2014) Polyelectrolyte films based on chitosan/olive oil and reinforced with cellulose nanocrystals. Carbohydr Polym 101:1018-26. https://doi.org/10.1016/j.carbpol.2013.10.046

Pereira AL et al (2014) Improvement of polyvinyl alcohol properties by adding nanocrystalline cellulose isolated from banana pseudostems. Carbohydr Polym 112:165-72. https://doi. org/10.1016/j.carbpol.2014.05.090

Perumal AB et al (2018) Development of polyvinyl alcohol/chitosan bio-nanocomposite films reinforced with cellulose nanocrystals isolated from rice straw. Appl Surf Sci 449:591-602. https:// doi.org/10.1016/j.apsusc.2018.01.022

Petroudy SRD et al (2018) Eco-friendly superabsorbent polymers based on carboxymethyl cellulose strengthened by TEMPOmediated oxidation wheat straw cellulose nanofiber. Carbohyd Polym 197:565-575. https://doi.org/10.1016/j.carbp ol.2018.06.008

Piermaria JA et al (2009) Films based on kefiran, an exopolysaccharide obtained from kefir grain: development and characterization. Food Hydrocolloids 23:684-690. https://doi. org/10.1016/j.foodhyd.2008.05.003

Plackett D et al (2010) Physical properties and morphology of films prepared from microfibrillated cellulose and microfibrillated cellulose in combination with amylopectin. J Appl Polym Sci 117:3601-3609. https://doi.org/10.1002/app.32254

Poole S, Simon M (1997) Technological trends, product design and the environment. Des Stud 18:237-248. https://doi. org/10.1016/S0142-694X(97)00003-3

Popescu MC (2017) Structure and sorption properties of CNC reinforced PVA films. Int J Biol Macromol 101:783-790. https:// doi.org/10.1016/j.ijbiomac.2017.03.168

Quoc L et al (2015) Effect of Xanthan gum Solution on the Preservation of Acerola (Malpighia glabra L.). Cercetari Agronomice in Moldova. 48:89-97. https://doi.org/10.1515/cerce-2015-0045

Rai M et al (2019) Smart nanopackaging for the enhancement of food shelf life. Environ Chem Lett 17:277-290. https://doi. org/10.1007/s10311-018-0794-8

Saito $\mathrm{T}$ et al (2009) Individualization of nano-sized plant cellulose fibrils by direct surface carboxylation using TEMPO catalyst under neutral conditions. Biomacromol 10:1992-1996. https ://doi.org/10.1021/bm900414t

Sánchez-García MD et al (2010) Morphology and water barrier properties of nanobiocomposites of $\kappa / 1$-hybrid carrageenan and cellulose nanowhiskers. J Agric Food Chem 58:12847-12857. https://doi.org/10.1021/jf102764e

Sanuja S et al (2015) Synthesis and characterization of zinc oxide-neem oil-chitosan bionanocomposite for food packaging application. Int J Biol Macromol 74:76-84. https://doi. org/10.1016/j.ijbiomac.2014.11.036

Savadekar NR et al (2012) Preparation of nano cellulose fibers and its application in kappa-carrageenan based film. Int $\mathrm{J}$ 
Biol Macromol 51:1008-13. https://doi.org/10.1016/j.ijbio mac.2012.08.014

Savadekar N, Mhaske S (2012) Synthesis of nano cellulose fibers and effect on thermoplastics starch based films. Carbohyd Polym 89:146-151. https://doi.org/10.1016/j.ijbiomac.2012.08.014

Saxena A, Ragauskas AJ (2009) Water transmission barrier properties of biodegradable films based on cellulosic whiskers and xylan. Carbohyd Polym 78:357-360. https://doi.org/10.1016/j.carbp ol.2009.03.039

Saxena A et al (2010) High oxygen nanocomposite barrier films based on xylan and nanocrystalline cellulose. Nano-Micro Letters. 2:235-241. https://doi.org/10.1007/BF03353849

Seydibeyoğlu MÖ, Oksman K (2008) Novel nanocomposites based on polyurethane and micro fibrillated cellulose. Composites Science and Technology. 68:908-914. https://doi.org/10.1016/j.comps citech.2007.08.008

Sfiligoj Smole M et al (2019) Cellulose nanofibres. surface properties of non-conventional cellulose fibres. Springer International Publishing, Cham, 2019, pp 61-71. https://doi.org/10.1007/9783-030-10407-8_5

Sharma A et al (2019) Commercial application of cellulose nano-composites-A review. Biotechnology Reports. 21:e00316. https://doi. org/10.1016/j.btre.2019.e00316

Siqueira G et al (2009) New process of chemical grafting of cellulose nanoparticles with a long chain isocyanate. Langmuir 26:402-411. https://doi.org/10.1021/la9028595

Soeta $\mathrm{H}$ et al (2015) Low-birefringent and highly tough nanocellulose-reinforced cellulose triacetate. ACS Appl Mater Interfaces 7:11041-6. https://doi.org/10.1021/acsami.5b02863

Sofla MRK et al (2016) A comparison of cellulose nanocrystals and cellulose nanofibres extracted from bagasse using acid and ball milling methods. Adv Nat Sci Nanosci Nanotechnol 7:035004. https://doi.org/10.1088/2043-6262/7/3/035004

Song Z et al (2014) Hydrophobic-modified nano-cellulose fiber/PLA biodegradable composites for lowering water vapor transmission rate (WVTR) of paper. Carbohydr Polym 111:442-8. https ://doi.org/10.1016/j.carbpol.2014.04.049

Soykeabkaew N et al (2012) Reinforcing potential of micro-and nano-sized fibers in the starch-based biocomposites. Composites Science and Technology. 72:845-852. https://doi. org/10.1016/j.compscitech.2012.02.015

Stelte W, Sanadi AR (2009) Preparation and characterization of cellulose nanofibers from two commercial hardwood and softwood pulps. Ind Eng Chem Res 48:11211-11219. https://doi. org/10.1021/ie9011672

Stuart MA et al (2010) Emerging applications of stimuli-responsive polymer materials. Nat Mater 9:101-113. https://doi. org/10.1038/nmat 2614

Svagan AJ et al (2007) Biomimetic polysaccharide nanocomposites of high cellulose content and high toughness. Biomacromol 8:2556-2563. https://doi.org/10.1016/j.compscitec h.2008.11.016

Svagan AJ et al (2009) Reduced water vapour sorption in cellulose nanocomposites with starch matrix. Composites Science and Technology. 69:500-506. https://doi.org/10.1016/j.compscitec h.2008.11.016

Swain SK, Mohanty F (2018) Polysaccharides-based bionanocomposites for food packaging applications. Bionanocomposites for packaging applications. Springer, Berlin, 2018, pp 191-208. https://doi.org/10.1007/978-3-319-67319-6_10

Tang A et al (2017) Biodegradable tissue engineering scaffolds based on nanocellulose/PLGA nanocomposite for NIH 3T3 cell cultivation. J Nanosci Nanotechnol 17:3888-3895. https://doi. org/10.1166/jnn.2017.13115
Teixeira EdM et al (2009) Cassava bagasse cellulose nanofibrils reinforced thermoplastic cassava starch. Carbohyd Polym 78:422431. https://doi.org/10.1016/j.carbpol.2009.04.034

Thakur VK (2013) Biomass-based biocomposites. Smithers Rapra. https://books.google.co.uk/books?id=jP81DwAAQB AJ\&lpg $=\mathrm{PP} 1 \& \mathrm{pg}=\mathrm{PP} 1 \# \mathrm{v}=$ onepage $\& \mathrm{q} \& \mathrm{f}=$ false

Thambiraj S, Shankaran DR (2017) Preparation and physicochemical characterization of cellulose nanocrystals from industrial waste cotton. Appl Surf Sci 412:405-416. https://doi.org/10.1016/j. apsusc.2017.03.272

Thomas B et al (2018) Nanocellulose, a versatile green platform: from biosources to materials and their applications. Chem Rev 118:11575-11625. https://doi.org/10.1021/acs.chemrev.7b00627

Tibolla $\mathrm{H}$ et al (2014) Cellulose nanofibers produced from banana peel by chemical and enzymatic treatment. LWT Food Sci Technol 59:1311-1318. https://doi.org/10.1016/j.1wt.2014.04.011

Tice P (2003) Packaging materials: 4. Polyethylene for food packaging applications, ILSI

Tofa TS et al (2019) Visible light photocatalytic degradation of microplastic residues with zinc oxide nanorods. Environ Chem Lett 17:1341-1346. https://doi.org/10.1007/s10311-019-00859-z

Vaia RA et al (1993) Synthesis and properties of two-dimensional nanostructures by direct intercalation of polymer melts in layered silicates. Chem Mater 5:1694-1696. https://doi.org/10.1021/ $\mathrm{cm} 00036 \mathrm{a} 004$

Vargas F et al (2012) Cellulosic pulps of cereal straws as raw material for the manufacture of ecological packaging. BioResources 7:4161-4170. http://ncsu.edu/bioresources

Wakai M, Almenar E (2015) Effect of the presence of montmorillonite on the solubility of whey protein isolate films in food model systems with different compositions and $\mathrm{pH}$. Food Hydrocoll 43:612-621. https://doi.org/10.1016/j.foodhyd.2014.07.022

Wang Y et al (2006) Effects of cellulose whiskers on properties of soy protein thermoplastics. Macromol Biosci. 6:524-31. https://doi. org/10.1002/mabi.200600034

Wang H-D et al (2015) Cellulose nanocrystals with $\mathrm{CO}_{2}$-switchable aggregation and redispersion properties. Cellulose 22:31053116. https://doi.org/10.1007/s10570-015-0690-3

Wang $\mathrm{Z}$ et al (2019) Microplastic contamination in freshwater: first observation in Lake Ulansuhai, Yellow River Basin, China. Environ Chem Lett 17:1821-1830. https://doi.org/10.1007/s1031 1-019-00888-8

Wu T et al (2014) Mechanical behavior of transparent nanofibrillar cellulose-chitosan nanocomposite films in dry and wet conditions. J Mech Behav Biomed Mater 32:279-286. https://doi. org/10.1016/j.jmbbm.2014.01.014

Xie H et al (2018) Recent strategies in preparation of cellulose nanocrystals and cellulose nanofibrils derived from raw cellulose materials. International Journal of Polymer Science. https://doi. org/10.1155/2018/7923068

Xiong Q et al (2018) Major trends and roadblocks in CFD-aided process intensification of biomass pyrolysis. Chem Eng Process Process Intensif 127:206-212. https://doi.org/10.1016/j. cep.2018.04.005

Xu X et al (2013) Cellulose nanocrystals vs. cellulose nanofibrils: a comparative study on their microstructures and effects as polymer reinforcing agents. ACS Appl Mater Interf 5:2999-3009. https://doi.org/10.1021/am302624t

Yakkan E et al (2018) Nanocellulose-polypropylene nanocomposites enhanced with coupling agent. Bartın Orman Fakültesi Dergisi 20:491-502. https://www.cabdirect.org/cabdirect/abstract/20193 014700

Yam KL, Lee DS (2012) Emerging food packaging technologies: principles and practice. Elsevier.www.sciencedirect.com/book/97818 45698096/emerging-food-packaging-technologies 
Yang W et al (2015) Structure and properties of biodegradable wheat gluten bionanocomposites containing lignin nanoparticles. Ind Crops Prod 74:348-356. https://doi.org/10.1016/j.indcr op.2015.05.032

Youssef AM et al (2015) Evaluation of bionanocomposites as packaging material on properties of soft white cheese during storage period. Carbohydr Polym 132:274-285. https://doi.org/10.1016/j. carbpol.2015.06.075

Youssef AM, El-Sayed SM (2018) Bionanocomposites materials for food packaging applications: concepts and future outlook. Carbohyd Polym 193:19-27. https://doi.org/10.1016/j.carbp ol.2018.03.088

Youssef AM et al (2019) Development of bionanocomposite materials and its use in coating of Ras cheese. Food Chem 270:467-475. https://doi.org/10.1016/j.foodchem.2018.07.114

Zainuddin SYZ et al (2013) Potential of using multiscale kenaf fibers as reinforcing filler in cassava starch-kenaf biocomposites. Carbohydr Polym 92:2299-2305. https://doi.org/10.1016/j.carbp ol.2012.11.106

Zhang C et al (2013) Effect of gelatin addition on properties of pullulan films. J Food Sci 78:C805-10. https://doi.org/10.111 1/j.1750-3841.2012.02925.x

Zhang C-C et al (2014) Tetrabromobisphenol A recovery from computer housing plastic by a new solvothermal process. Environ Chem Lett 12:347-352. https://doi.org/10.1007/s1031 1-014-0452-8

Zhang HC et al (1997) Environmentally conscious design and manufacturing: a state-of-the-art survey. Journal of manufacturing systems. 16:352-371. https://doi.org/10.1016/ S0278-6125(97)88465-8

Zhao Y et al (2017) Cellulose nanofibers from softwood, hardwood, and tunicate: preparation-structure-film performance interrelation. ACS Appl Mater Interfaces 9:13508-13519. https://doi. org/10.1021/acsami.7b01738

Zhong $\mathrm{H}$ et al (2019) CFD modeling of the effects of particle shrinkage and intra-particle heat conduction on biomass fast pyrolysis. Renew Energy 141:236-245. https://doi.org/10.1016/j.renen e.2019.04.006

Zhong $\mathrm{H}$ et al (2020) CFD-based reduced-order modeling of fluidized-bed biomass fast pyrolysis using artificial neural network. Renew Energy 152:613-626. https://doi.org/10.1016/j.renen e.2020.01.057

Zhu JY et al (2011) Integrated production of nano-fibrillated cellulose and cellulosic biofuel (ethanol) by enzymatic fractionation of wood fibers. Green Chem 13:1339-1344. https://doi. org/10.1039/C1GC15103G

Zinge C, Kandasubramanian B (2020) Nanocellulose based Biodegradable Polymers. Eur Polymer J. https://doi.org/10.1016/j.eurpo lymj.2020.109758

Publisher's Note Springer Nature remains neutral with regard to jurisdictional claims in published maps and institutional affiliations. 